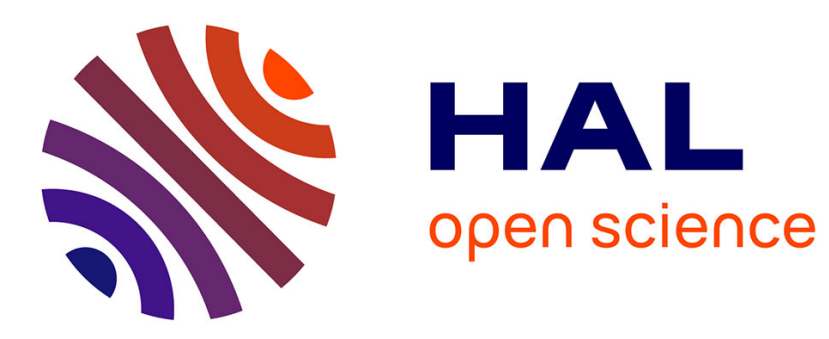

\title{
Brittle Fracture Theory Describes the Onset of Frictional Motion
}

Ilya Svetlizky, Elsa Bayart, Jay Fineberg

\section{To cite this version:}

Ilya Svetlizky, Elsa Bayart, Jay Fineberg. Brittle Fracture Theory Describes the Onset of Frictional Motion. Annual Review of Condensed Matter Physics, 2019, 10 (1), pp.253-273. 10.1146/annurevconmatphys-031218-013327 . hal-03026026

\section{HAL Id: hal-03026026 \\ https://hal.science/hal-03026026}

Submitted on 5 Jan 2021

HAL is a multi-disciplinary open access archive for the deposit and dissemination of scientific research documents, whether they are published or not. The documents may come from teaching and research institutions in France or abroad, or from public or private research centers.
L'archive ouverte pluridisciplinaire HAL, est destinée au dépôt et à la diffusion de documents scientifiques de niveau recherche, publiés ou non, émanant des établissements d'enseignement et de recherche français ou étrangers, des laboratoires publics ou privés. 


\title{
Brittle Fracture Theory Describes the Onset of Frictional Motion
}

\author{
Ilya Svetlizky, Elsa Bayart and Jay Fineberg \\ The Racah Institute of Physics, The Hebrew University of Jerusalem, Jerusalem 91904, Israel; \\ email: jay@mail.huji.ac.il
}

\begin{abstract}
Contacting bodies subjected to sufficiently large applied shear will undergo frictional sliding. The onset of this motion is mediated by dynamically propagating fronts, akin to earthquakes, that rupture the discrete contacts that form the interface separating the bodies. Macroscopic motion commences only after these ruptures have traversed the entire interface. Comparison of measured rupture dynamics to the detailed predictions of fracture mechanics reveals that the propagation dynamics, dissipative properties, radiation and arrest of these "laboratory earthquakes" are in excellent quantitative agreement to the predictions of the theory of brittle fracture. Thus, interface fracture replaces the idea of a characteristic static friction coefficient as a description of the onset of friction. This fracture-based description of friction additionally provides a fundamental description of earthquake dynamics and arrest.
\end{abstract}

\section{Contents}

1 INTRODUCTION 1

1.1 Single Degree of Freedom Representation of Friction . . . . . . . 2

1.2 Spatio-temporal Dynamics at the Onset of Frictional Motion . . 4

2 FRICTIONAL RUPTURE FRONTS - EXPERIMENTS AND MODELING 5

2.1 Singular Elastic Fields Drive Frictional Rupture Fronts . . . . . . 5

2.2 Equation of Motion of Frictional Rupture Fronts . . . . . . . . 9

2.3 Rupture Arrest . . . . . . . . . . . . . . . . . . . . 11

2.4 High Amplitude Stress-Wave Radiation - Non-Singular Contri-

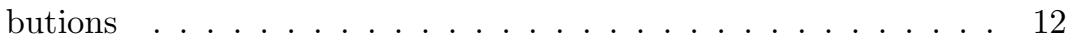

2.5 Regularization of the Singular Fields . . . . . . . . . . 13

3 CONCLUSIONS AND OPEN QUESTIONS 


\section{INTRODUCTION}

Frictional resistance has puzzled mankind for thousands of years. One reason is its enormous importance. Frictional forces will stop our cars - as well as keep them on the road. Extensive efforts have been directed towards understanding friction across scales; from single nanometric contacts $[48,68,141]$ to natural faults and earthquakes $[29,96,103,134]$. While descriptions of friction date back to at least the early Egyptians $[57,116]$, the foundations for the modern study of friction were laid by Leonardo da Vinci, whose early experiments suggested that the onset of frictional motion is predicted by the ratio of shear to normal forces acting on contacting bodies. The simplicity of this description has been challenged by recent experiments. Detailed measurements have revealed that the onset of sliding is mediated by complex spatio-temporal dynamics that take place at the contacting interface that separates sliding bodies. Motion is initiated when the ensemble of micro-contacts, that make up a rough frictional interface, are broken by means of propagating rupture fronts. These rupture fronts, which are akin to earthquakes, may approach the speed of sound. Macroscopic sliding of two bodies commences only after such a front has traversed the entire frictional interface. These rupture fronts, which can be mapped to cracks, couple dynamics at time and length scales that are separated by many orders of magnitude. The interplay of these is the key to understanding friction.

This review focuses on the dynamics of frictional rupture fronts at the onset of motion. Our aim is to both show how the friction problem is related to the fracture dynamics and present experimental evidence that validates this 'fracture mechanics' framework. To this end, we intentionally omit numerous important topics in the study of friction such as contact mechanics and effects of roughness [72,83,109,113,115,146], wear [2], microscopic models of frictional interfaces $[3,22,116]$ and stability of frictional sliding $[22,39,126]$. Furthermore, we simplify our discussion to sub-Rayleigh rupture fronts propagating along an interface separating identical materials. We will therefore not include in this review recent contributions in our understanding of supershear ruptures and rupture propagating along bimaterial interfaces. The structure of this review is as follows. After briefly reviewing single degree of freedom descriptions of friction, we will sketch, in sec. 2.1, the fracture mechanics theoretical framework that replaces this simplistic view of friction, when the spatial degrees of freedom along an extended frictional interface are properly accounted for. After demonstrating that the elastic fields at the tip of propagating ruptures are very well described by the universal singular solutions originally developed to describe brittle shear cracks (sec. 2.1), we will show that the classical equation of motion derived for brittle shear cracks perfectly describes the acceleration (sec. 2.2) and arrest (sec. 2.3) of frictional ruptures (laboratory earthquakes). Moreover, we will demonstrate how this quantitative framework can be used to explore the elusive properties of both the hitherto "hidden" interface. In sec. 2.4 we go on to demonstrate how non-singular contributions capture the unique form

of stress-wave radiation resulting from rapid rupture acceleration. Finally, we discuss how the singular fields at a rupture tip are regularized (sec.2.5). 


\subsection{Single Degree of Freedom Representation of Friction}

Let us consider two contacting bodies that are pressed together by an external normal force $F_{N}$ and subjected to a slowly increasing shear loading (Fig. 1a top). This system will eventually undergo slip-stick motion; alternating start-stop motion. In the stick phase, the interface separating the two bodies resists the relative motion of the bodies and $F_{S}$ increases slowly with the applied loading. At the onset of motion, $F_{S}$ rapidly decreases as the relative motion (slip) of the blocks commences; the sliding bodies accelerate away from the points where $F_{S}$ is applied (Fig. 1b) reducing its value rapidly. Typically, frictional resistance at the onset of motion is larger than the resistance during sustained sliding. Both are commonly thought to be proportional to the applied normal force where the proportionality factors, $\mu_{s}$ and $\mu_{d}$, are respectively defined as the 'static' and 'dynamic' friction coefficients. In this rather simple description, typically referred to as Amonton-Coulomb friction laws, friction coefficients depend solely on the material properties, which are independent of the loading configuration, the geometry of the bodies and the nominal area of the frictional interface.

In the 1950's Bowden and Tabor [35] laid the foundations for understanding Amonton-Coulomb friction laws. They realized that the real area of contact $A$ formed by rough contacting surfaces is substantially smaller than the nominal contact area, $A_{n o m}$, as it is composed of myriad discrete microscopic contacts (Fig. 1a bottom). The applied nominal normal pressure, $\sigma=F_{N} / A_{n o m}$, is balanced by the mean pressure at these contacts. The real pressure at these contacts is therefore huge, $F_{N} / A$, often reaching the material hardness, $\sigma_{H}$. If an ideal plastic model is considered, one expects that

$$
A / A_{n o m} \sim \sigma / \sigma_{H}
$$

as was demonstrated for some transparent materials [50]. The proportionality between $A$ and $\sigma$ is not, however, unique to situations where the touching asperities deform plasticly and may occur even under fully elastic deformation if a reasonable distribution of the asperities height and radius is taken into account $[72]$.

In the next step, frictional resistance per nominal unit area $\tau$ is related to the shear strength of the micro-contacts $\tau_{s}$ by

$$
\tau=\tau_{s} \cdot A / A_{\text {nom }}
$$

By combining both equations we get $\mu_{s}=\tau_{s} / \sigma_{H}$, which encapsulates the salient features of Amonton-Coulomb friction.

There have been a number of modifications to the Amonton-Coulomb friction laws. For completeness, we will briefly mention some of these. Pioneering experiments by Rabinowicz [119] revealed two significant corrections; healing and slip rate dependence. $\mu_{s}$ was shown to increase logarithmically with time when surfaces are held in stationary contact under applied normal load. This "healing" enables the static friction to recover from its lower dynamic value attained during sliding. Slip rate dependence characterized by logarithmic velocity weakening of $\mu_{d}$ for steady sliding was also observed for slow slip velocities 
$v<1 \mathrm{~mm} / \mathrm{s}$. Finally, the transition between one value of friction to another was found to be accompanied by a critical slip distance. This phenomenology is rather general and has been observed for metals [119], rock [51,96,132] and polymer glasses $[21,22,30]$.

All of this phenomenology can be incorporated in rate-and-state formulations of friction. The starting points are Eqs. 1 and 2 where an instantaneous rate dependence of the micro-contact shear strength, $\tau_{s}=\tau_{s}(v)$, is introduced. Generally, $\tau_{s}(v)$ is an increasing function of $v$ [17]. The history dependence and the evolution of the contact area are given by $A(\phi)$, where $\phi$ is defined as a state variable that is typically interpreted as the "contact life time". Setting $\phi=t$, for instance, captures the logarithmic increase of $A$ with contact time (aging) of pressed materials $[28,49,100]$ and therefore describes the "healing" of $\mu_{s}$ [30]. Note, however, that recent experiments indicate that $A$ exhibits rich healing effects which cannot be fully captured by a single state variable [53]. Extensive efforts have been invested in formulating evolution laws for $\phi$ that typically have the form $\dot{\phi}=f(\phi, v)[31,96,100,101]$. Evolution laws are necessary to capture the time and slip history of both the evolution of friction with $v$ as well as the dynamics of interface healing.

Typically, the above corrections to Amontons-Coulomb friction are both small and are observed for conditions of low slip rates $(v<1 \mathrm{~mm} / \mathrm{s})$ and modest displacements $(<1 \mathrm{~cm})$. At these rates, the heat generated at the contacts can diffuse away appreciably over a contact lifetime $\left(d_{c} / v\right.$, where $d_{c}$ is a typical contact size), resulting in a rather small temperature increases. Motivated by the high seismic slip rates characteristic of large earthquakes, rotary shear apparatuses have recently tested much higher slip rates $(\sim 1 \mathrm{~m} / \mathrm{s})$. At sufficiently high slip rates, experiments in rock have demonstrated extreme reductions of the frictional resistance. These have been associated with a variety of different mechanisms that include flash heating (local melting) of the contacts $[71,111,125]$ powder lubrication $[45,123]$ of the ground rock ('gouge') that is trapped within the frictional interfaces, triggering of mechanically and thermally activated chemical reactions [70], and, eventually, melting [42]. For an extensive review of this class of experiments, see Di-Toro et al. [47].

\subsection{Spatio-temporal Dynamics at the Onset of Frictional Motion}

The underlying premise in the friction studies previously discussed is that the sliding bodies are entirely rigid. Hence, their spatial degrees of freedom can be neglected and their relative motion can be represented by a single degree of freedom. The combined stiffness of the experimental apparatus and the elasticity of the bodies surrounding the interface, in this view, is often modeled by a single effective "spring". The applied shear force (deformation of the spring) essentially provides a measure of the friction force, if inertia is neglected.

In realistic cases, however, these assumptions are not satisfied and frictional motion entails rich and complex spatio-temporal dynamics. One such example is the onset of frictional motion. As materials are both flexible and deformable, 
rather than rigid, it is not realistic to assume that sliding will initiate along the entire frictional interface instantaneously and simultaneously at each contacting point. Instead, motion (slip) initiates locally and overall sliding of the bodies commences only after propagating crack-like rupture fronts, that separate the sticking and sliding spatial regions, traverse the interface. Figures $1 \mathrm{~d} \&$ e present typical examples of both slowly $\left(C_{f} \sim 0.1 C_{R}\right)$ and rapidly $\left(C_{f} \sim C_{R}\right)$ propagating ruptures, respectively, where $C_{f}$ and $C_{R}$ are respectively the speed of the fronts and the Rayeigh wave speed (the asymptotic velocity of singular shear cracks). The propagating fronts are visualized by high-speed spatial measurements of $A$ [130]. In these examples, ruptures nucleate at $x \approx 0$, accelerate in the positive $x$ direction, and leave in their wake a significantly reduced $A$.

Ignoring these rupture fronts by modeling the motion of spatially extended sliding bodies by their center of mass, a single degree of freedom, may, therefore, result in erroneous conclusions. Ben-David and Fineberg [27] demonstrated that the macroscopically measured static friction coefficient, $\mu_{s}=F_{S} / F_{N}$, is not only a material constant but also may vary systematically with controlled variation of the external loading configuration. These variations were tightly linked to changes in the rupture dynamics. Furthermore, Fig. 1 demonstrates that $\mu_{s}$ may significantly vary even within the same stick-slip sequence, with lower $\mu_{s}$ values associated with nucleation of slower ruptures. The whole concept of a "static friction coefficient" is therefore insufficient to determine the onset of global frictional slip. This is exemplified by large reported variations in measurements of $\mu_{s}$ [120] (up to a factor of 2) under ostensibly identical conditions. The criterion for global slip, instead, is intimately related to the question of what determines rupture nucleation.

The importance of the spatio-temporal dynamics of these rapidly propagating fronts is further highlighted if one considers the hundreds of $\mathrm{km}$ long natural faults that separate tectonic plates. The slow motion of tectonic plates over hundreds of years results in a gradual increase of their stored elastic energy. The release of this energy occurs through rapidly propagating rupture fronts that generate the strong ground motion that we equate with earthquakes [134]. The intimate relation between earthquakes and laboratory stick-slip instability, as

was recognized by Brace and Byerlee [36], has triggered extensive experimental studies of crack-like dynamics along frictional interfaces [75, 76, 103,107, 150].

\section{FRICTIONAL RUPTURE FRONTS - EX- PERIMENTS AND MODELING}

\subsection{Singular Elastic Fields Drive Frictional Rupture Fronts}

We will now show that the rupture fronts, such as those described in Fig. 1, are essentially shear cracks. We will limit our discussion to 'sub-Rayleigh' ruptures $\left(C_{f}<C_{R}\right)$ that propagate within an interface separating identical materials. To proceed, we will first briefly review the basic concepts of Linear Elastic Fracture Mechanics (LEFM), the theoretical framework that describes crack propagation. 
The dynamic behavior of deformed linearly elastic materials can be described by two wave equations, one for longitudinal waves (with a characteristic wave speed $C_{L}$ ) and one for shear waves (with characteristic wave speed $C_{S}$ ) $[41,62]$. Mathematically, a propagating crack in a two dimensional solid (see Fig. 2a) is introduced by traction free boundary conditions at the crack faces i.e. $\sigma_{x y}=$ $\sigma_{y y}=0$. These boundary conditions essentially couple both wave equations. The in-plane opening mode of tensile crack deformation (mode I - $\sigma_{y y}$ ), the inplane shearing mode (mode II $-\sigma_{x y}$ ), and the anti-plane shearing mode (mode III $\left.-\sigma_{y z}\right)$ can be treated separately. Solving the complete time-dependent boundary value problem is a formidable task. A solution for a steadily moving crack, propagating at speeds below $C_{S}$, can be obtained, however, in the form of an asymptotic expansion in powers of $r^{n / 2}$, where $r$ is the distance from the crack tip and $n$ is an integer. In the close vicinity of a crack tip, stresses are described by a singular term (with integrable energy) that dominates nonsingular contributions

$$
\sigma_{i j}=\sum_{\alpha=\mathrm{I}, \mathrm{II}, \mathrm{III}} \frac{K_{\alpha}}{\sqrt{2 \pi r}} \Sigma_{i j}^{\alpha}\left(\theta, C_{f}\right)
$$

where $(r, \theta)$ are polar coordinates with respect to the crack tip. $\Sigma_{i j}^{\alpha}$ are known universal functions. Here, $\alpha$ labels the different fracture modes and $C_{f}$ is the instantaneous crack velocity. The scalar quantities $K_{\alpha}$ are commonly called the stress intensity factors. $K_{\alpha}$ are determined by the outer boundary conditions (loading conditions) as well as crack's profile, length $l$, history and instantaneous dynamics [62]. $\sigma_{i j}$ are linearly related to the strain field $\varepsilon_{i j}$ via Hook's law $\sigma_{i j}=2 \mu\left[\varepsilon_{i j}+\left(1 / 2 k^{2}-1\right) \varepsilon_{l l} \delta_{i j}\right]$ where $\mu$ is the shear modulus and $k=C_{S} / C_{L}$ [41]. This square-root singularity of the elastic fields, which essentially defines brittle fracture, is universal in the sense that its form does not depend on the geometry and outer boundary conditions.

It is interesting that shear-driven (mode II) fracture is generally considered to be impossible in bulk isotropic materials because it is believed that a crack will rotate under imposed shear so as to fracture under pure tension [59]. The highly anisotropic conditions embodied in the case of the weak interface plane that defines a frictional interface, constrain the crack to this plane and thereby provide one case where mode II fracture may indeed be realized. This important case is the focus of this review. The problem of crack path selection, one of the major unsolved fracture mechanics problems, is circumvented here since only mode II straight cracks are considered.

With the elastic fields in hand, the energy flux through any closed contour surrounding the crack tip can be calculated. It can be shown that within the singular region (where the $K$-fields dominate) $K_{\text {II }}$ determines the energy flux per unit crack advance, $G_{\mathrm{II}}$ :

$$
G_{\mathrm{II}}=\frac{K_{\mathrm{II}}^{2}}{4 \mu\left(1-k^{2}\right)} f_{\mathrm{II}}\left(C_{f}, k\right)
$$

where $f_{\mathrm{II}}\left(C_{f}, k\right)$ is a known function $[41,62]$ that is fairly constant for low velocities and diverges as $C_{f}$ approaches the Rayleigh wave speed, $C_{R}$ (note 
that $\left.C_{R}<C_{S}\right) . C_{R}$ is the limiting value of $C_{f}$ so long as the point singularity embodied in Eq 3 holds (see sec. 2.2).

The fracture energy $\Gamma$ is defined as the energy dissipated per unit of newly created surface area. $\Gamma$ incorporates all unknown dissipation mechanisms. Its calculation from first principles is very challenging and thus one relies on experimental measurements to determine its value. For a crack to propagate, the elastic energy flowing into the crack tip must equal the energy dissipated by creating new surface. Hence, the energy balance condition $\Gamma=G_{\mathrm{II}}$ must hold.

A crucial difference exists between real cracks and frictional ruptures. While crack faces are stress free (the material is broken), within the wake of frictional ruptures the bodies are always in partial contact $\left(\sigma_{y y}^{0}(x) \neq 0\right)$ and the frictional resistance, $\tau_{r}(x)$, opposes sliding. Using the linearity of the governing equations, however, the problem of a frictional rupture front, propagating within an interface separating identical materials, can be mapped to the stress-free conditions that define the mode II crack problem [110]. Thus, stresses in a vicinity of a frictional rupture tip are therefore predicted to be:

$$
\sigma_{i j}=\frac{K_{\mathrm{II}}}{\sqrt{2 \pi r}} \Sigma_{i j}^{\mathrm{II}}\left(\theta, C_{f}\right)+\left[\begin{array}{cc}
\sigma_{x x}^{0} & \tau_{r} \\
\tau_{r} & \sigma_{y y}^{0}
\end{array}\right] .
$$

Note however, that when friction depends on the sliding velocity, for example, linear viscous friction, a new form of singularity may emerge [38]. We also note that ruptures propagating along (bimaterial) interfaces that separate bodies with different elastic properties $[5,7,135,148,152]$ will have different dynamics.

The infinite stresses at the rupture tip are naturally regularized in some vicinity of the rupture tip, which is called the process or cohesive zone. The region where the square-root singular form dominates, therefore, should be interpreted as an "intermediate asymptotic" region separating the "inner" scales of dissipation from the "outer" region where non-singular contributions can not be neglected (Fig. 2a). This assumption is typically coined "small scale yielding" [124].

Is Eq. 5 together with the assumption of small scale yielding valid for frictional rupture fronts? Recent experiments [137] have shed light on this question. The complete 2D strain tensor $\varepsilon_{i j}$ was measured slightly above the frictional interface formed by two acrylic (PMMA) plates (see Fig. 1a and Fig. 2a). Figures $2 \mathrm{c}$ and $2 \mathrm{~d}$ show measured $\varepsilon_{i j}$ for the slow and fast rupture events presented in Fig. 1d and Fig. 1e, respectively. As $C_{f}$ increases, all $\varepsilon_{i j}$ amplitudes grow significantly and strain oscillations are strongly amplified. Comparison with the square-root singular form predicted by LEFM shows that for slow ruptures all of the measured strain components $\varepsilon_{i j}$ agree well with the strains corresponding to equation 5 (black lines), where the only free parameter used was the value of $K_{\mathrm{II}}$. Because the strain measurements were, necessarily, displaced from the interface, each $\varepsilon_{i j}$ measurement involved both radial and angular variations (see Fig. $2 \mathrm{~b}) . K_{\mathrm{II}}$ is related uniquely to $\Gamma$ for each $C_{f}$ by using Eq. 4 and energy balance $G_{\mathrm{II}}=\Gamma$. These measurements therefore provided the explicit value of $\Gamma$ at the interface. 
Figure $2 \mathrm{~d}$ demonstrates that for the same constant value of $\Gamma$ nearly all of the characteristic features of $\varepsilon_{i j}$ observed at higher rupture velocities are also well described by the $1 / \sqrt{r}$ form. For example, the violent high-amplitude strain oscillations that occur when the rupture tip passes beneath the measurement point (Fig. 2b) are due to the singular nature of $\Sigma_{i j}^{\mathrm{II}}\left(\theta, C_{f}\right)$ as $C_{f} \rightarrow C_{R}$. In fact, ref. [137] concluded that with a single input value of $\Gamma$, the theory quantitatively describes the strain variations throughout the entire sub-Rayleigh velocity range $0.1 C_{R}<C_{f}<0.99 C_{R}$. Figure 2d, top, however, shows that Eq. 5 clearly fails to describe $\varepsilon_{x y}$ ahead of the rupture tip $\left(x-x_{t i p}>0\right)$. This apparent "discrepancy" with the singular solution was shown to be due to non-singular contributions [139]. Its importance will be discussed in section 2.4.

The ability of LEFM to describe the strain fields in the rupture tip vicinity is entirely general. The characteristic signature of the $1 / \sqrt{r}$ form, (for example the strong oscillations in $\varepsilon_{x y}$ in Fig. 2d) have been observed in a variety of different brittle materials, homalite (Fig. 4 in ref. [129]), granite (Fig. 1 in ref. [106], Fig. 10 in ref. [104] and Fig. 1 in ref. [98]) and Indian meta-gabbro (Fig. 9 in ref. [154]).

The generality of LEFM was further demonstrated in ref. [23], where the onset of motion in the boundary lubrication regime was explored. In this regime, the contacting surfaces are covered by a thin lubricant layer, and the discrete asperities still bear the entire normal load, as they are not entirely immersed in the fluid layer. As in dry friction (Fig. 1 and Fig. 2), each sliding event is preceded by propagating rupture fronts that break the solid contacts forming the interface. Figure 3(a) shows explicitly that the strain field variations in the rupture tip vicinity are, again, well described by LEFM. Hence, these ruptures are true brittle shear cracks. Surprisingly, while the lubricated interface reduces the dynamic and static friction relative to the dry interface, the inferred value of $\Gamma$, for the same applied normal load, can be order of magnitude greater than for the same non-lubricated interfaces. It is interesting that, as Fig. 3b shows, the values of $\Gamma$ are independent of the lubricant viscosity, although highly dependent on the lubricant's chemical composition.

What determines the value of $\Gamma$ ? Let's first consider dry interfaces. Whereas the bulk fracture energy, $\Gamma_{\text {Bulk }}$ is a material property $\left(\approx 2,000 \mathrm{~J} / \mathrm{m}^{2}\right.$ for PMMA), the interface value, $\Gamma$, linearly increases with $\sigma_{y y}$ as shown in Fig. (3)b. As $\sigma_{y y} \propto A$ (Eq. 1), $\Gamma$ essentially accounts for the change in contact area necessary for a rupture to propagate. In fact, when the sparseness of the contacts [49] $\left(A<<A_{n o m}\right)$ was accounted for, the measured values of $\Gamma$ were indeed found [137] to be consistent with $\Gamma_{B u l k}$. This suggests that significant plastic deformation (the major contribution to $\Gamma_{B u l k}$ in PMMA) should also take place within the contacting asperities.

Why does $\Gamma$ increase with the addition of a lubricant? The proportionality of $\Gamma$ in this regime with $\sigma_{y y}$ (hence $A$ ) suggests that the enhancement of $\Gamma$ by the lubricant layer only takes place at contacts. The enhancement mechanism and its dependence on the chemical composition of the lubricant is both intriguing and not completely understood [23]. While at the microscopic level these results are puzzling, these findings highlight the fact that the fracture 
mechanics description of frictional motion provides a new way to probe the otherwise hidden and complex dynamics of the all-important interface. Without the "microscope" that the fracture mechanical analysis of the strength of the frictional layer described in this section provides, we would be entirely blind to the detailed properties of this critical region. This (often) micron-thick layer both possesses wholly different properties than those of the bulk material and determines the strength of the entire macroscopic system.

\subsection{Equation of Motion of Frictional Rupture Fronts}

How fast can rupture fronts propagate? A wide range of rupture front velocities within laboratory experiments have been observed. These range from slow ruptures $[26,102,105,130,137]$ propagating at a small fraction of the Rayleigh wave speed, $C_{R}$, to ruptures that asymptotically approach $C_{R}$. Typical subRayleigh examples are shown in Fig. 1d and Fig. 1e and their velocities are plotted in Fig. 4d. Under suitable conditions rupture fronts may also surpass the shear wave speed, $C_{S}$, and approach the longitudinal speed of sound, $C_{L}[26,85,99,111,127,139,151,153]$.

Experiments by Ben-David et al. [26] first revealed that rupture fronts increase their speed with increased shear to normal stress ratios. Motivated by fracture mechanics, it was suggested that this stress ratio reflects the balance between the potential energy available, prior to rupture, and the energy required to rupture the interface. Later experiments in rock [112], simulations [77, 122, 142-144] and analytical 1D models [15] confirmed these observations.

Once the LEFM predictions (Fig. 2) for the functional forms of the near-tip stress fields were verified [137], it became possible to test the theory's quantitative predictions for sub-Rayleigh rupture dynamics. These are provided by the energy balance $\Gamma=G_{\mathrm{II}}$ criterion, that provides an implicit equation of motion for shear crack. Quantitative predictions can be made if one is able to explicitly calculate $G_{\text {II }}$, or equivalently $K_{\text {II }}$ (see Eq. 4). Such calculations for a general loading configuration are extremely challenging. Freund, in seminal work [60-62], considered a semi-infinite crack in an unbounded medium subjected to time independent loading. The crack is initially at rest and then moves at $C_{f}<C_{R}$. The stress intensity factor for this type of loading can be explicitly calculated. $K_{\mathrm{II}}$ has the following decomposition $K_{\mathrm{II}}\left(l, C_{f}, \Delta \sigma_{x y}\right)=$ $\kappa_{\mathrm{II}}\left(C_{f}\right) K_{\mathrm{II}}^{S}\left(l, \Delta \sigma_{x y}\right)$. Here $\kappa_{\mathrm{II}}\left(C_{f}\right)$ is a known [62] universal dynamic function that depends solely on the instantaneous value of $C_{f} . K_{\mathrm{II}}^{S}\left(l, \Delta \sigma_{x y}\right)$, the static/equilibrium stress intensity factor, depends on the instantaneous crack length, $l$, and incorporates all of the information about the loading through the dynamic stress drop $\Delta \sigma_{x y}=\sigma_{x y}^{0}-\tau_{r}$. Here, $\sigma_{x y}^{0}$ is the initial stress level, prior to the rupture's arrival, while $\tau_{r}$ is the residual frictional resistance (see Fig. 2.2a).

Following Eq. 4 and the decomposition of $K_{\text {II }}, G$ can now be decomposed into $G_{\mathrm{II}}=G_{\mathrm{II}}^{S}\left(l, \Delta \sigma_{x y}\right) g_{\mathrm{II}}\left(C_{f}\right)$ where $G_{\mathrm{II}}^{S}\left(l, \Delta \sigma_{x y}\right)$, the static energy release rate, can be explicitly calculated once $K_{\mathrm{II}}^{S}\left(l, \Delta \sigma_{x y}\right)$ is known. For example, under 
uniformly applied shear, where $\Delta \sigma_{x y}$ is independent of $x, G_{\mathrm{II}}^{S}$ is an increasing function of the crack length; $G_{\mathrm{II}}^{S} \propto\left(\Delta \sigma_{x y}\right)^{2} l / \mu$. The dynamic correction to the energy flux is given by $g_{\mathrm{II}}\left(C_{f}\right)$, a universal monotonically decreasing function of $C_{f}$ that is unity at the limit $C_{f} \rightarrow 0$ and zero at $C_{f}=C_{R}$ (see [62] for details). Under these conditions, the equation of motion, $C_{f}(l)$, for arbitrary stress configurations is implicitly given by

$$
\Gamma=G_{\mathrm{II}}=G_{\mathrm{II}}^{S}\left(l, \Delta \sigma_{x y}\right) g_{\mathrm{II}}\left(C_{f}\right) .
$$

Two main consequences can be directly deduced from Eq. 6 and the properties of $g_{\mathrm{II}}\left(C_{f}\right)$. (1) Crack propagation is energetically possible if and only if $G_{\mathrm{II}}^{S}\left(l, \Delta \sigma_{x y}\right) \geq \Gamma$. In particular, under uniformly applied shear, crack propagation is possible only above a critical crack length, termed the Griffith length, $l_{c} \sim \mu \Gamma /\left(\Delta \sigma_{x y}\right)^{2}$. (2) $C_{R}$ is the limiting crack velocity which can only be reached asymptotically when $G_{\mathrm{II}}^{S}\left(l, \Delta \sigma_{x y}\right) \rightarrow \infty$. In particular, under these conditions, cracks will accelerate asymptotically to $C_{R}$ as $l \rightarrow \infty$.

In Ref. [138] a quantitative test of Eq. 6 was conducted for both unlubricated (dry) and boundary lubricated interfaces. These results are shown in Fig. 4. Figure 4c presents the measured profiles of $\Gamma$ together with the profiles of $G_{\mathrm{II}}^{S}\left(l, \Delta \sigma_{x y}\right)$ that were calculated using the measured fields $\Delta \sigma_{x y}(x)$ shown in Fig. $4 \mathrm{~b}$. Note that while $\Gamma$ was obtained by direct measurement of the dynamic singular fields (in the "near-field") at the rupture tip, $G_{\mathrm{II}}^{S}$ was calculated using solely the stress drops, $\Delta \sigma_{x y}$, ahead of the rupture tip ("far field" stresses). Figures $4 c \& d$ demonstrate that slow ruptures (e.g. blue line) propagate when the static elastic energy released by a unit advance of the crack is nearly balanced by the dissipated energy, $G_{\mathrm{II}}^{S} \approx \Gamma$. Loading conditions that result in significant excess elastic energies, $G_{\mathrm{II}}^{S} \gg \Gamma$, generate rapid acceleration to $C_{R}$ (e.g. green line). All of the rupture velocities $C_{f}(l)$, when plotted with respect to $G_{\mathrm{II}}^{S}\left(l, \Delta \sigma_{x y}\right) / \Gamma$, collapse onto the precise functional form predicted by Eq. 6 (Figure 4e). These experiments explicitly demonstrate that, with no adjustable parameters, the classical equation of motion for brittle shear cracks provides an excellent quantitative description of the velocity evolution of frictional rupture fronts.

Slow ruptures, $C_{f} \ll C_{R}$, observed in laboratory experiments have drawn much recent attention due to accumulating numbers of observed slow earthquakes [114]. In geoscience, slow earthquakes have often been regarded as an entirely different entity than the more traditionally observed rapid $\left(C_{f} \sim C_{R}\right)$ earthquakes. As a result, the driving mechanisms for slow earthquakes are still very much under debate. It has been suggested that slow ruptures emerge as a result of a crossover from velocity-weakening friction at slow slip to velocitystrengthening at higher slip rates $[15,16,33,81]$. Alternatively, slow ruptures have been observed in stochastic multi-scale simulations [143,144]. While nontrivial friction laws might well be an important mechanism for stabilizing slow ruptures, measurements such as those presented in Fig. 4 suggest that slow ruptures may simply emerge from any nontrivial stress distribution that would retain a nearly "static" energy balance defined by $G_{\mathrm{II}}^{S} \approx \Gamma$, along the propagation path. 
The experiments in Fig. 4 describe rupture dynamics that result from a relatively broad range of imposed shear stress levels prior to rupture initiation - hence each took place for very different values of the applied shear force, $F_{S}$ (see Fig. 1b). Each of these experiments, however, was performed with the same imposed value of $F_{N}$. This observation underlines the fact that there is really no characteristic value of $\mu_{s}$. In fact, it was the shear stress at which each rupture nucleated that determined the explicit value of $\mu_{s}$. This stick-slip sequence is an excellent demonstration that frictional interfaces can be either stable or unstable for the same loading conditions, in stark contradiction to the picture of frictional onset implied by 'single degree of freedom' descriptions of friction described in sec. 1.1. Instability of a frictional interface is triggered by the nucleation process - the process by which an initial "crack" emerges from within a rough interface.

\subsection{Rupture Arrest}

Frictional ruptures can arrest well before spanning an entire interface. These types of arrested frictional ruptures were first observed in experiments by $\mathrm{Ru}-$ binstein et al. [131] and a comprehensive review of interface rupture arrest can be found in ref. [24]. Here we briefly describe the main physics of how interface ruptures arrest.

Two typical examples of such arrested ruptures are presented in Fig. 5c. Rupture arrest can, for example, result from inhomogeneous stress distributions along the interface. In laboratory experiments, such distributions are often observed if shear forces $F_{S}$ are preferentially applied at one edge of a sample (see Fig. 5a-inset). Under such loading conditions, a succession of arrested ruptures of increasing lengths are typically observed (e.g. examples A and B in Fig. 5), well before $F_{S}$ reaches the threshold for the overall stick-slip motion of the blocks (e.g. example $\mathrm{C}$ in Fig. 5).

Rupture arrest has been observed numerically $[82,121,122]$ and variety of models have been designed to describe the dynamics of precursory ruptures in frictional systems. Aimed at reproducing nucleation and arrest, these include minimalistic one-dimensional (1D) [94] and scalar [140] models, discrete contact descriptions [37,142] and rate-and-state friction laws [18]. While these models were able to reproduce arrested ruptures, they provided no explicit general predictions of where and how arrest occurs in real systems.

Recently, Kammer et al. [78] demonstrated that fracture mechanics can be used to predict the rupture arrest locations observed in ref. [131]. In the LEFM framework, crack arrest will take place if the energy flux to the tip of a quasistatically propagating crack (see definition in section 2.2) is insufficient to overcome the fracture energy; $G_{\mathrm{II}}^{S}\left(l, \Delta \sigma_{x y}\right)<\Gamma$. This criterion for rupture arrest was explicitly verified in experiments [25], in which a number of different loading conditions and system geometries were considered. In these experiments there were no free parameters; the experimental "ingredients" required by Eq. $6, \Gamma$ and $G_{\mathrm{II}}^{S}\left(l, \Delta \sigma_{x y}\right)$, were either directly measured or calculated by means of direct measurements of $\Delta \sigma_{x y}(l)$. Calculated profiles $G_{\mathrm{II}}^{S}\left(l, \Delta \sigma_{x y}\right)$ for the typical 
stick-slip sequence described in Fig. 5a are presented in Fig. 5b. As the figure demonstrates, the predicted arrest locations, $l_{\text {predicted }}$, for each event are indeed the precise locations where $G_{\mathrm{II}}^{S}\left(l, \Delta \sigma_{x y}\right)<\Gamma$ (denoted by the solid circles in Figs. 5b,c). Figure 5c (bottom) demonstrates that, in general, $l_{\text {predicted }}$ is in excellent agreement with measured arrest lengths, $l_{\text {measured }}$, obtained from the contact area measurements.

It is critical to note that while rupture nucleation, as discussed in the previous section, is a necessary condition for the onset of motion it is insufficient to determine global sliding of the blocks. In this sense, overall frictional slip may occur only if a rupture front reaches the system size, $L$. Only when $l_{\text {predicted }}=L$ will the entire interface be ruptured and will overall slip of the contacting bodies ensue.

While the results described above are generally relevant for any frictional interface, they are especially important to understand the essentially unresolved question of what determines the size of an earthquake [108]. In fact, fracture mechanics had been used to relate the spatial stress heterogeneities to earthquake sizes distribution [8] and recently [66] was successfully implemented to predict the arrest of injection-induced earthquakes. Eq. 6 demonstrates that earthquake magnitudes can be determined by:

- Nonuniform values of $\Gamma$ along an interface; at any location where $G_{\mathrm{II}}^{S}\left(l, \Delta \sigma_{x y}\right)<$ $\Gamma$ an earthquake should immediately arrest.

- The effects of nonuniform stress profiles; this is an integral effect, as the value of $G_{\mathrm{II}}^{S}\left(l, \Delta \sigma_{x y}\right)$ is determined by a weighted integral of the stresses $\Delta \sigma_{x y}(l)$ distributed along an interface.

\subsection{High Amplitude Stress-Wave Radiation - Non-Singular Contributions}

It has long been suggested that nonsteady processes such as the rapid rupture velocity variation during the nucleation or arrest phases result in the generation of stress-wave radiation [56,93]. In the study of earthquakes, understanding the source mechanism of those waves is of primary importance. Laboratory experiments, therefore, have provided a unique opportunity to shed light on this subject.

A typical example of a rupture front, asymptotically accelerating to rupture velocities, $C_{f} \rightarrow C_{R}$, is presented in Fig. 6a (adapted from [139]). Figure 6b presents measurements of shear stresses $\sigma_{x y}$, at two distinct time steps, $t_{1}$ and $t_{2}$ (as noted in Fig. 6a). It is evident that prominent peaks in $\sigma_{x y}$ precede the rupture tip arrival. Analysis of peak arrival times (e.g., the black circles in Fig. 6a) reveals that they propagate at $C_{S}$ and, therefore, progressively distance themselves from the rupture tips that created them. Extrapolating the space-time peak trajectories to the intersection point with the rupture trajectory implies 
that the initiation of this radiated stress wave originates within the latter stages of the accelerating phase.

In crack tip vicinities, all stress (strain) components have the universal $1 / \sqrt{r}$ form, as this singular contribution dominates the near-tip stress fields in brittle fracture. These stress peaks, as was mentioned in section 2.1, are not described by this $1 / \sqrt{r}$ form (black line in Fig. 6b). A general description of these radiated stress-waves does not exist. Any such analytical description must go beyond the singular contributions to the stress fields. Such full solutions of generally nonsteady dynamic crack problems are extremely difficult to obtain [84]. In some simplified cases, however, analytical solutions for accelerating shear cracks are available and have provided much insight $[41,56,62,93]$. One such solution, which was derived both for tension [40] and shear [44], describes bilaterally expanding cracks that initiate with zero initial length and propagate at a constant velocity $\left(C_{f}<C_{R}\right)$ under uniformly applied remote shear stresses (see also $[41,62])$. In this problem, there is no characteristic time or length scale so self-similar propagating solutions can be found.

The resulting normalized shear stress on the interface $(y=0)$ is plotted in Fig. 6c. This solution reveals a singular propagating crack tip that is preceded by a sharp and relatively localized shear stress peak that propagates at $C_{S}$. The form and amplitude of this stress peak is generally considered to be an upper bound for the realistic stress-wave radiation of smoothly accelerating ruptures.

Figure $6 \mathrm{~b}$ shows that this self-similar solution of an expanding shear rupture can describe the measured shear stress rather well. In particular, this solution can capture both the initial shear loading, $\sigma_{x y}^{0}$, as well as the propagating shear stress peak far before the rupture tip arrival. This particular solution demonstrates the importance of the nonsingular contributions to strains at finite distances from the rupture front tip [63]. Furthermore, the apparent "discrepancy" with the singular solution (observed in Fig. 6b and the upper panel of Fig. 2d) is not a simple technical issue of accounting for nonsingular contributions to the singular description, but actually is a nontrivial radiated wave that possesses a life (and extensive history) of its own. Analytical solutions [56,93] and accompanying finite element simulations [139] demonstrate that these radiated shear stress peaks have a characteristic near-field signature: high-amplitude radiation (comparable to the dynamic stress drop) that is both localized and strongly focused in the direction of rupture propagation.

The comparison with the self-similar solution highlights the underlying physical picture. Rapid rupture front acceleration (mimicked by the infinite acceleration in the self-similar problem) results in radiation having the form of a localized shear stress peak propagating at $C_{S}$. The measurements of $A(x, t)$ presented in Fig. 6a furthermore reveal the sudden nucleation $(x \approx 155 \mathrm{~mm})$ of a secondary supershear rupture front (propagating at $C_{f}>C_{S}$ ), which surpasses the classic "speed limit", $C_{R}$, for singular cracks. The synchronized measurements of $\sigma_{x y}$ and $A(x, t)$ provide direct evidence that the supershear rupture was triggered by the arrival of the shear stress peak. This nucleation mechanism was first postulated in ref. [44]. Later numerical work [10] found that a sufficiently strong shear stress peak can overcome interfacial strength 
and nucleate a daughter crack that could propagate at supershear velocities. Supershear ruptures have been observed along natural fault planes [34,55], in laboratory experiments $[26,85,99,111,127,151,154]$ and have been extensively investigated by numerical simulations $[1,4,20,43,58,65,67,87,88,118]$. A further description, however, is beyond the scope of this review.

\subsection{Regularization of the Singular Fields}

In the previous section we briefly described the role of nonsingular "far field" contributions to the singular description of LEFM. As we discussed in section 2.1 , the singular $1 / \sqrt{r}$ region is interpreted as an "intermediate asymptotic"; the region that connects the process/cohesive zone in which dissipation takes place to the "outer" region, where non-singular contributions cannot be neglected (see the schematic view in Fig. 2a). In section 2.1 the "intermediate" $1 / \sqrt{r}$ singular region was described. From the singular fields one can directly characterize the interface by means of the fracture energy, $\Gamma$. In general, however, very little is known about how the singular fields are regularized within the process zone. How different materials regularize the rupture tip singularities is an interesting and rather important question, as the properties of different materials in this elusive region entirely determine interface strengths. These questions are central to numerous (rather disparate) applications that range from the effects of additives on frictional wear to how natural fault properties affect earthquake dynamics. As interface conditions are extreme, interface properties may have little in common with a material's bulk equilibrium properties (as we saw for the case of boundary layer lubrication).

Regularization of rupture tip singularities is not expected to be universal, as various dissipative processes may take place. Simple models, however, can be used to capture the essential physical mechanisms that take place near the rupture tip. The simplest regularization model was implemented by Barenblatt [19], Dugdale [54], Ida [74] and others, who showed that the singularity of the stress fields can be eliminated by postulating cohesive forces working across the weak plane. In this approach, which is typically termed "slip-weakening" [110], weakening of the local frictional resistance, $\tau$, is initiated once the shear stress has reached a finite peak strength, $\tau_{p}$. Subsequently, $\tau(d)$ gradually decreases with the local slip, $d$, until reaching the dynamic friction level, $\tau_{r}$. This occurs at a critical slip distance, $d_{c}$. The fracture energy is defined as the energy

dissipated during weakening, $\Gamma=\int_{0}^{d_{c}}\left(\tau(d)-\tau_{r}\right) \mathrm{d} d$. Note that $\Gamma$ does not account for the dissipation due to residual level of friction $\tau_{r}$. Due to the linearity of the governing equations, subtracting away $\tau_{r}$ (and its associated dissipation) enables the mapping of the friction problem to fracture (see Eq. 5).

The simplicity of slip-weakening models makes them extremely useful. They have been extensively used to simulate frictional rupture fronts in a number of contexts which include the superhear transition $[11,12,32,87,88,90]$, off-fault damage $[13,14,153]$ and 3D rupture propagation [149].

Analytically, however, it is often more convenient to use cohesive zone models $[110,117,133]$, where shear stresses gradually decrease from $\tau_{p}$ to $\tau_{r}$ with the 
spatial position, according to a prescribed spatial stress profile, $\tau(x)=\left(\tau_{p}-\right.$ $\left.\tau_{r}\right) \cdot \widetilde{\tau}\left(x / x_{c}\right)+\tau_{r}$ [see Fig. 7a (inset)]. Here $x=0$ is the rupture tip and $x_{c}$ is defined to be the cohesive zone size. Small scale yielding is assumed and the problem is closed by the universal boundary conditions dictated by the singular $K$ - fields, i.e., far ahead of the crack tip the solution matches the square-root singular form, $\sigma_{x y}\left(x>>x_{c}, y=0\right) \rightarrow K_{\mathrm{II}} / \sqrt{2 \pi x}$ [133]. Therefore, $\left(\tau_{p}-\tau_{r}\right), x_{c}$ and $\Gamma$ are related through Eq. 4 and

$$
K_{\mathrm{II}}=\left(\tau_{p}-\tau_{r}\right) \cdot \sqrt{x_{c}} \cdot \sqrt{\frac{2}{\pi}} \cdot \int_{-\infty}^{0} \frac{\widetilde{\tau}(\xi)}{\sqrt{-\xi}} d \xi
$$

Once the solution is obtained, the equivalent slip-weakening constitutive law $\tau(d)$, can be calculated.

These models are often criticized as being too simple since they do not take into account the rate and history dependence of the frictional resistance. As discussed in sec. 1.1, an intensive and on-going effort is directed towards formulation of constitutive laws which endeavors to address these effects.

Direct measurements of the constitutive evolution law of friction are impeded by the singular nature of the fields. On the other hand, measurements of the real contact area, as performed in the reviewed experiments, shed light on how these singular fields are regularized, since these measurements, by definition, take place on the interface. Figures 7 a demonstrate that $A(x, t)$ indeed decreases gradually behind the propagating rupture tip, in clear contrast to idealized singular cracks for which an abrupt reduction $A(x, t)$ is expected. The length scale over which $A(x, t)$ is reduced [137] provides an estimate of the cohesive zone size, $x_{c}$. Figure $7 \mathrm{a} \& \mathrm{~b}$ demonstrate that $x_{c}$ is not constant, but systematically contracts with increasing $C_{f}$. It was shown [137] that this effective "Lorenz" contraction is predicted by LEFM (Eq. 4 and Eq. 7) and is described by $x_{c}\left(C_{f}\right)=x_{c}\left(C_{f}=0\right) / f_{\mathrm{II}}\left(C_{f}, k\right)$, where $f_{\mathrm{II}}\left(C_{f}, k\right)$ (note that $f_{\mathrm{II}} \rightarrow 0$ as $C_{f} \rightarrow C_{R}$ ) is the same universal function that appears in Eq. 4 (see Fig. 7b).

Knowledge of $\Gamma, x_{c}$ and the assumption of the simplest cohesive zone model, enable us to estimate elusive but long sought after constitutive parameters that characterize the dissipative processes and material properties at the extreme conditions that take place near the rupture tip. These include the peak shear strength $\tau_{p}$ (Fig. 7c,d - top), maximal slip velocity $2 \dot{u}_{x}$ (Fig. $7 \mathrm{c}, \mathrm{d}$ - bottom), and critical slip distance, $d_{c}$.

Figure 7 reveals that measurements performed slightly above the frictional interface do not reflect any of the cohesive zone dynamics or properties. This is especially true for $C_{f} \rightarrow C_{R}$, since $x_{c} \rightarrow 0$ in this regime. Measurements at finite distances from a frictional interface should, therefore, be interpreted with extreme caution as the divergence of the near-tip fields may result in erroneous conclusions. Credible measurements of the interface properties are particularly, challenging as they can only be obtained if measurements are performed at distances from the rupture tip that are much smaller than $x_{c}$. It is progressively harder to meet this requirement as $x_{c}$ contracts with the rupture velocity. Combined measurements of the real area of contact, slip and stresses at the interface 
and their evolution is certainly an important research direction, if one would like to assess interface properties under friction.

\section{CONCLUSIONS AND OPEN QUESTIONS}

As we have demonstrated above, the transition from stick to slip is mediated by propagating rupture fronts. These ruptures couple dynamics at time and length scales that are separated by many orders of magnitude and determine whether macroscopic motion will ensue or not.

Although rupture fronts have long been considered to have much in common with propagating cracks, there had been little direct experimental evidence that quantitative universal predictions of linear elastic fracture mechanics really describe frictional failure. This brief review has summarized recent experimental results that have established the extensive applicability of brittle fracture theory to our understanding of both frictional rupture dynamics and to earthquake dynamics, an important but particular case. In particular, we have seen that:

- The elastic fields in the vicinity of both rapidly propagating and slow frictional rupture tips are extremely well-described by the universal squareroot singular solutions originally developed to describe brittle shear cracks.

- The singularity and dynamics of frictional ruptures are identical for both dry and boundary lubricated interfaces.

- The singular fields provide a quantitative measure of the fracture energy, $\Gamma$, that is needed to advance a rupture.

- The dynamic behavior and arrest of frictional ruptures is entirely described by the classical fracture mechanics description of shear crack dynamics. Both of these have important implications for a fundamental understanding of earthquake dynamics. This 'simple' description describes both extremely slow and rapid rupture fronts. The only physical quantities needed to differentiate between these extremes is the amount of elastic energy stored in the system prior to rupture nucleation and, of course, the distribution of the fracture energy along the interface.

The general "simplicity" and beauty of this approach should be emphasized. Once $\Gamma$, the sole free parameter that encapsulates the dissipative processes at the rupture tip has been measured, rupture propagation, acceleration and arrest are entirely predicted by the balance with the energy flux to the crack tip. This energy flux depends solely on the difference between the initial shear stress and the dynamic frictional resistance along the interface. Whereas a specific material (with both dry and boundary-lubricated interfaces) has been considered in this review, the results are general so long as several necessary conditions are satisfied. First, a small scale yielding approximation should apply; a region in the rupture tip's vicinity should exist where $\sigma \sim 1 / \sqrt{r}$. Second, there should be no significant rate dependence of the frictional resistance within the rupture tail. 
This condition enables one to map the frictional interface to stress free boundary conditions. Third, the particular analysis that we have used is valid for all times prior to the arrival of waves reflected from a sample's far boundaries back to the rupture tip. Once this occurs, fracture mechanics should still describe rupture evolution, but a different (time-dependent) analysis of the singular fields will be necessary (see e.g. [69,95] for examples in tensile fracture).

Seismic inversions of earthquakes [73] and some laboratory experiments $[89,91,92]$ have implied that ruptures may also be "pulse-like", where frictional slip ceases shortly behind the rupture front. This is in contrast to the "cracklike" modes considered here, where the slip velocity is not confined to a finite zone. Pulse-like behavior can occur when an interface is formed by two bodies with different elastic properties $[7,135]$ (forming a "bimaterial interface") or for materials that undergo enhanced velocity weakening (stronger than logarithmic) at high slip rates $[7,46,65,91,155]$. The form of the cohesive law governing the interface strength is quite important in this respect; for example, slip-weakening friction laws were unable to reproduce this rich phenomenology $[91,155]$. While in PMMA slip-pulse modes haven't been observed, there is experimental evidence for slip-pulse modes in a different brittle plastic, Homalite [89, 91, 92]. One key difference may be the behavior of the residual stress, $\tau_{r}$, behind the rupture tip. In PMMA $\tau_{r}$ only mildly varies with the slip velocity [136] whereas in Homolite strong velocity weakening has been reported [129].

The propagation criterion outlined in Section 2.1 is a necessary condition for the onset of frictional motion. The onset of frictional motion, however, also requires rupture nucleation. We have shown that, once nucleation takes place, fracture mechanics will quantitatively describe ensuing rupture dynamics and/or arrest. It is however, unclear how to predict when and via what mechanisms will nucleation take place. For the Griffith criterion for rupture propagation to apply, for example, a singular "seed" crack must first exist. Understanding the spontaneous formation of such a seed crack from within a rough frictional interface is a fundamental question of great importance. Despite a large number of interesting theoretical $[6,9,16,52,79,80,103,128,145,147]$ and experimental $[64,86,97,102]$ efforts, it is still, however, not very well understood. From an experimental perspective there is a striking paucity of direct experimental observations of how nucleation takes place. Local measurements, within the nucleation region and cohesive zone, of stresses, slip and contact area are very much needed.

\section{DISCLOSURE STATEMENT}

The authors are not aware of any affiliations, memberships, funding, or financial holdings that might be perceived as affecting the objectivity of this review. 


\section{ACKNOWLEDGMENTS}

We acknowledge the support of both the US-Israel Binational Science Foundation (Grant No. 2016950), and Israel Science Foundation (Grant No. 1523/15).

\section{References}

[1] F. F. Abraham and H. J. Gao. How fast can cracks propagate? Physical Review Letters, 84(14):3113-3116, 2000.

[2] Ramin Aghababaei, Derek H. Warner, and Jean-Francois Molinari. Critical length scale controls adhesive wear mechanisms. Nature Communications, 7:11816, Jun 2016.

[3] Einat Aharonov and Christopher H. Scholz. A physics-based rock friction constitutive law: Steady state friction. Journal of Geophysical Research: Solid Earth, 2018. 2016JB013829.

[4] Gabriele Albertini and David S. Kammer. Off-fault heterogeneities promote supershear transition of dynamic mode ii cracks. Journal of Geophysical Research: Solid Earth, 122(8):6625-6641, 2017.

[5] Michael Aldam, Yohai Bar-Sinai, Ilya Svetlizky, Efim A. Brener, Jay Fineberg, and Eran Bouchbinder. Frictional sliding without geometrical reflection symmetry. Phys. Rev. X, 6:041023, Oct 2016.

[6] Michael Aldam, Marc Weikamp, Robert Spatschek, Efim A. Brener, and Eran Bouchbinder. Critical nucleation length for accelerating frictional slip. Geophysical Research Letters, 44(22):11,390-11,398, 2017. 2017GL074939.

[7] J.-P. Ampuero and Y. Ben-Zion. Cracks, pulses and macroscopic asymmetry of dynamic rupture on a bimaterial interface with velocity-weakening friction. Geophysical Journal International, 173(2):674-692, 2008.

[8] J.-P. Ampuero, J. Ripperger, and P. M. Mai. Properties of Dynamic Earthquake Ruptures with Heterogeneous Stress Drop, pages 255-261. American Geophysical Union, 2006.

[9] Jean-Paul Ampuero and Allan M. Rubin. Earthquake nucleation on rate and state faults - aging and slip laws. Journal of Geophysical Research: Solid Earth, 113(B1), 2008.

[10] D. J. Andrews. A numerical study of tectonic stress release by underground explosions. Bulletin of the Seismological Society of America, 63(4):1375-1391, 1973.

[11] D. J. Andrews. Rupture velocity of plane strain shear cracks. Journal of Geophysical Research-Solid Earth, 81:5679, 1976. 
[12] D. J. Andrews. Dynamic plane-strain shear rupture with a slip-weakening friction law calculated by a boundary integral method. Bulletin of the Seismological Society of America, 75(1):1-21, 1985.

[13] D. J. Andrews. Rupture dynamics with energy loss outside the slip zone. Journal of Geophysical Research: Solid Earth, 110(B1), 2005. B01307.

[14] D.J. Andrews. Ground motion hazard from supershear rupture. Tectonophysics, 493(3):216 - 221, 2010.

[15] Yohai Bar-Sinai, Efim A. Brener, and Eran Bouchbinder. Slow rupture of frictional interfaces. Geophysical Research Letters, 39(3):L03308, 2012.

[16] Yohai Bar-Sinai, Robert Spatschek, Efim A. Brener, and Eran Bouchbinder. Instabilities at frictional interfaces: Creep patches, nucleation, and rupture fronts. Physical Review E, 88(6):060403, 2013.

[17] Yohai Bar-Sinai, Robert Spatschek, Efim A. Brener, and Eran Bouchbinder. On the velocity-strengthening behavior of dry friction. Journal of Geophysical Research: Solid Earth, 119(3):1738-1748, 2014.

[18] Yohai Bar-Sinai, Robert Spatschek, Efim A. Brener, and Eran Bouchbinder. Velocity-strengthening friction significantly affects interfacial dynamics, strength and dissipation. Scientific Reports, 5:7841, 2015.

[19] G.I. Barenblatt. The Mathematical Theory of Equilibrium Cracks in Brittle Fracture. Advances in Applied Mechanics. 7 edition, 1962.

[20] Fabian Barras, Philippe H. Geubelle, and Jean-Fran çois Molinari. Interplay between process zone and material heterogeneities for dynamic cracks. Phys. Rev. Lett., 119:144101, Oct 2017.

[21] T. Baumberger, P. Berthoud, and C. Caroli. Physical analysis of the stateand rate-dependent friction law. ii. dynamic friction. Physical Review B, 60:3928-3939, Aug 1999.

[22] T. Baumberger and C. Caroli. Solid friction from stick-slip down to pinning and aging. Advances in Physics, 55(3-4):279-348, 2006.

[23] E. Bayart, I. Svetlizky, and J. Fineberg. Slippery but tough: The rapid fracture of lubricated frictional interfaces. Physical Review Letters., 116:194301, May 2016.

[24] E. Bayart, I. Svetlizky, and J. Fineberg. Rupture dynamics of heterogeneous frictional interfaces. Journal of Geophysical Research, 123, 2018.

[25] Elsa Bayart, Ilya Svetlizky, and Jay Fineberg. Fracture mechanics determine the lengths of interface ruptures that mediate frictional motion. Nature Physics, 12(2):166-170, 2016. 
[26] Oded Ben-David, Gil Cohen, and Jay Fineberg. The dynamics of the onset of frictional slip. Science, 330(6001):211-214, 2010.

[27] Oded Ben-David and Jay Fineberg. Static friction coefficient is not a material constant. Physical Review Letters, 106:254301, Jun 2011.

[28] Oded Ben-David, Shmuel M. Rubinstein, and Jay Fineberg. Slip-stick and the evolution of frictional strength. Nature, 463(7277):76-79, 2010.

[29] Yehuda Ben-Zion. Collective behavior of earthquakes and faults: Continuum-discrete transitions, progressive evolutionary changes, and different dynamic regimes. Reviews of Geophysics, 46(4), 2008. RG4006.

[30] P. Berthoud, T. Baumberger, C. G'Sell, and J.-M. Hiver. Physical analysis of the state- and rate-dependent friction law: Static friction. Physical Review B, 59:14313-14327, Jun 1999.

[31] Pathikrit Bhattacharya, Allan M. Rubin, Elsa Bayart, Heather M. Savage, and Chris Marone. Critical evaluation of state evolution laws in rate and state friction: Fitting large velocity steps in simulated fault gouge with time-, slip-, and stress-dependent constitutive laws. Journal of Geophysical Research: Solid Earth, 120(9):6365-6385, 2015.

[32] A. Bizzarri, M. Cocco, D. J. Andrews, and E. Boschi. Solving the dynamic rupture problem with different numerical approaches and constitutive laws. Geophysical Journal International, 144(3):656, 2001.

[33] Eran Bouchbinder, Efim A. Brener, Itay Barel, and Michael Urbakh. Slow cracklike dynamics at the onset of frictional sliding. Phys. Rev. Lett., 107:235501, Nov 2011.

[34] M. Bouchon and M. Vallee. Observation of long supershear rupture during the magnitude 8.1 kunlunshan earthquake. Science, 301(5634):824-826, 2003.

[35] F. P. Bowden and D. Tabor. The Friction and Lubrication of Solids. Oxford Univ. Press, New York, 2 edition, 2001.

[36] W. F. Brace and J. D. Byerlee. Stick-slip as a mechanism for earthquakes. Science, 153(3739):990-992, 1966.

[37] O. M. Braun, I. Barel, and M. Urbakh. Dynamics of transition from static to kinetic friction. Physical Review Letters, 103(19):194301, 2009.

[38] E. A. Brener and V. I. Marchenko. Frictional shear cracks. Jetp Letters, 76(4):211-214, 2002.

[39] Efim A. Brener, Marc Weikamp, Robert Spatschek, Yohai Bar-Sinai, and Eran Bouchbinder. Dynamic instabilities of frictional sliding at a bimaterial interface. Journal of the Mechanics and Physics of Solids, 89:149$173,2016$. 
[40] K. Bertram Broberg. The propagation of a brittle crack. Arkiv Fysik, 18(2):159-92, 1960.

[41] K. Bertram Broberg. Cracks and Fracture. Academic Press, San Diego, 1999.

[42] Kevin M. Brown and Yuri Fialko. Melt welt mechanism of extreme weakening of gabbro at seismic slip rates. Nature, 488(7413):638-641, 2012.

[43] Lucile Bruhat, Zijun Fang, and Eric M. Dunham. Rupture complexity and the supershear transition on rough faults. Journal of Geophysical Research: Solid Earth, 121(1):210-224, 2016.

[44] R. Burridge. Admissible speeds for plane-strain self-similar shear cracks with friction but lacking cohesion. Geophysical Journal of the Royal Astronomical Society, 35(4):439-455, 1973.

[45] J. C. Chang, D. A. Lockner, and Z. Reches. Rapid acceleration leads to rapid weakening in earthquake-like laboratory experiments. Science, 338(6103):101-105, 2012.

[46] Alain Cochard and Raúl Madariaga. Dynamic faulting under ratedependent friction. pure and applied geophysics, 142(3):419-445, 1994.

[47] G. Di Toro, R. Han, T. Hirose, N. De Paola, S. Nielsen, K. Mizoguchi, F. Ferri, M. Cocco, and T. Shimamoto. Fault lubrication during earthquakes. Nature, 471(7339):494-498, 2011.

[48] Martin Dienwiebel, Gertjan S. Verhoeven, Namboodiri Pradeep, Joost W. M. Frenken, Jennifer A. Heimberg, and Henny W. Zandbergen. Superlubricity of graphite. Phys. Rev. Lett., 92:126101, Mar 2004.

[49] J. H. Dieterich and B. D. Kilgore. Direct observation of frictional contacts new insights for state-dependent properties. Pure and Applied Geophysics, 143(1-3):283-302, 1994.

[50] J. H. Dieterich and B. D. Kilgore. Imaging surface contacts: Power law contact distributions and contact stresses in quartz, calcite, glass and acrylic plastic. Tectonophysics, 256(1-4):219-239, 1996.

[51] James H. Dieterich. Modeling of rock friction: 1. experimental results and constitutive equations. Journal of Geophysical Research: Solid Earth, 84(B5):2161-2168, 1979.

[52] James H. Dieterich. Earthquake nucleation on faults with rate-and statedependent strength. Tectonophysics, 211(1):115 - 134, 1992.

[53] S Dillavou and SM Rubinstein. arXiv:1801.00011 [cond-mat.soft], 2018.

[54] D.S. Dugdale. Yielding of steel sheets containing slits. Journal of the Mechanics and Physics of Solids, 8(2):100 - 104, 1960. 
[55] E. M. Dunham and R. J. Archuleta. Evidence for a supershear transient during the 2002 denali fault earthquake. Bulletin of the Seismological Society of America, 94(6):S256-S268, 2004.

[56] Eric M. Dunham. Conditions governing the occurrence of supershear ruptures under slip-weakening friction. Journal of Geophysical Research: Solid Earth, 112(B7):B07302, 2007.

[57] A. Fall, B. Weber, M. Pakpour, N. Lenoir, N. Shahidzadeh, J. Fiscina, C. Wagner, and D. Bonn. Sliding friction on wet and dry sand. Phys. Rev. Lett., 112:175502, Apr 2014.

[58] G. Festa and J.-P. Vilotte. Influence of the rupture initiation on the intersonic transition: Crack-like versus pulse-like modes. Geophysical Research Letters, 33(15):L15320, 2006.

[59] J. Fineberg and M. Marder. Instability in dynamic fracture. Physics Reports-Review Section of Physics Letters, 313(1-2):2-108, 1999.

[60] L. B. Freund. Crack propagation in an elastic solid subjected to general loading. i. constant rate of extension. Journal of the Mechanics and Physics of Solids, 20(3):129-40, 1972.

[61] L. B. Freund. Crack propagation in an elastic solid subjected to general loading - ii. non-uniform rate of extension. Journal of the Mechanics and Physics of Solids, 20(3):141 - 152, 1972.

[62] L. B. Freund. Dynamic Fracture Mechanics. Cambridge, New York, 1990.

[63] L. B. Freund and A. J. Rosakis. The structure of the near-tip field during transient elastodynamic crack growth. Journal of the Mechanics and Physics of Solids, 40(3):699-719, 1992.

[64] Eiichi Fukuyama, Kotoyo Tsuchida, Hironori Kawakata, Futoshi Yamashita, Kazuo Mizoguchi, and Shiqing Xu. Spatiotemporal complexity of 2-d rupture nucleation process observed by direct monitoring during large-scale biaxial rock friction experiments. Tectonophysics, 2017.

[65] A.-A. Gabriel, J.-P. Ampuero, L. A. Dalguer, and P. M. Mai. The transition of dynamic rupture styles in elastic media under velocity-weakening friction. Journal of Geophysical Research: Solid Earth, 117(B9):B09311, 2012 .

[66] Martin Galis, Jean Paul Ampuero, P. Martin Mai, and Frédéric Cappa. Induced seismicity provides insight into why earthquake ruptures stop. Science Advances, 3(12), 2017.

[67] P. H. Geubelle and D. V. Kubair. Intersonic crack propagation in homogeneous media under shear-dominated loading: numerical analysis. Journal of the Mechanics and Physics of Solids, 49(3):571-587, 2001. 
[68] E. Gnecco, R. Bennewitz, T. Gyalog, Ch. Loppacher, M. Bammerlin, E. Meyer, and H.-J. Güntherodt. Velocity dependence of atomic friction. Phys. Rev. Lett., 84:1172-1175, Feb 2000.

[69] Tamar Goldman, Ariel Livne, and Jay Fineberg. Acquisition of inertia by a moving crack. Physical Review Letters, 104:114301, Mar 2010.

[70] David L. Goldsby and Terry E. Tullis. Low frictional strength of quartz rocks at subseismic slip rates. Geophysical Research Letters, 29(17):25-125-4, 2002. 1844.

[71] David L. Goldsby and Terry E. Tullis. Flash heating leads to low frictional strength of crustal rocks at earthquake slip rates. Science, 334(6053):216$218,2011$.

[72] J. A. Greenwood and J. B. P. Williamson. Contact of nominally flat surfaces. Proceedings of the Royal Society of London A: Mathematical, Physical and Engineering Sciences, 295(1442):300-319, 1966.

[73] T. H. Heaton. Evidence for and implications of self-healing pulses of slip in earthquake rupture. Physics of the Earth and Planetary Interiors, 64(1):1-20, 1990.

[74] Y. Ida. Cohesive force across tip of a longitudinal-shear crack and griffiths specific surface energy. Journal of Geophysical Research, 77(20):3796, 1972 .

[75] T Johnson and C. H. Scholz. Dynamic properties of stick-slip friction of rock. Journal of Geophysical Research, 81(5):881-888, 1976.

[76] Tracy Johnson, Francis T. Wu, and Christopher H. Scholz. Source parameters for stick-slip and for earthquakes. Science, 179(4070):278-280, 1973.

[77] D. S. Kammer, V. A. Yastrebov, P. Spijker, and J. F. Molinari. On the propagation of slip fronts at frictional interfaces. Tribology Letters, 48(1):27-32, 2012.

[78] David S. Kammer, Mathilde Radiguet, Jean-Paul Ampuero, and JeanFrançois Molinari. Linear elastic fracture mechanics predicts the propagation distance of frictional slip. Tribology Letters, 57(3):1-10, 2015.

[79] Y. Kaneko and J.-P. Ampuero. A mechanism for preseismic steady rupture fronts observed in laboratory experiments. Geophysical Research Letters, 38(21):L21307, 2011.

[80] Yoshihiro Kaneko, Stefan B. Nielsen, and Brett M. Carpenter. The onset of laboratory earthquakes explained by nucleating rupture on a rate-andstate fault. Journal of Geophysical Research: Solid Earth, 121(8):60716091, 2016. 
[81] Bryan M. Kaproth and C. Marone. Slow earthquakes, preseismic velocity changes, and the origin of slow frictional stick-slip. Science, 341(6151):1229-1232, 2013.

[82] Yu Katano, Ken Nakano, Michio Otsuki, and Hiroshi Matsukawa. Novel friction law for the static friction force based on local precursor slipping. Scientific Reports, 4:6324 EP -, Sep 2014. Article.

[83] L. Kogut and I. Etsion. Elastic-plastic contact analysis of a sphere and a rigid flat. Journal of Applied Mechanics, 69(5):657-662, Aug 2002.

[84] B. V. Kostrov. On the crack propagation with variable velocity. International Journal of Fracture, 11(1):47-56, Feb 1975.

[85] S. Latour, T. Gallot, S. Catheline, C. Voisin, F. Renard, E. Larose, and M. Campillo. Ultrafast ultrasonic imaging of dynamic sliding friction in soft solids: The slow slip and the super-shear regimes. EPL (Europhysics Letters), 96(5):59003, 2011.

[86] S. Latour, A. Schubnel, S. Nielsen, R. Madariaga, and S. Vinciguerra. Characterization of nucleation during laboratory earthquakes. Geophysical Research Letters, 40(19):5064-5069, 2013.

[87] Chao Liu, Andrea Bizzarri, and Shamita Das. Progression of spontaneous in-plane shear faults from sub-rayleigh to compressional wave rupture speeds. Journal of Geophysical Research: Solid Earth, 119(11):8331-8345, 2014.

[88] Y. Liu and N. Lapusta. Transition of mode ii cracks from sub-rayleigh to intersonic speeds in the presence of favorable heterogeneity. Journal of the Mechanics and Physics of Solids, 56:25-50, 2008.

[89] X. Lu, N. Lapusta, and A. J. Rosakis. Pulse-like and crack-like ruptures in experiments mimicking crustal earthquakes. Proceedings of the National Academy of Sciences of the United States of America, 104(48):1893118936, 2007.

[90] X. Lu, N. Lapusta, and A. J. Rosakis. Analysis of supershear transition regimes in rupture experiments: the effect of nucleation conditions and friction parameters. Geophysical Journal International, 177(2):717-732, 2009 .

[91] Xiao Lu, Nadia Lapusta, and Ares J. Rosakis. Pulse-like and crack-like dynamic shear ruptures on frictional interfaces: experimental evidence, numerical modeling, and implications. International Journal of Fracture, 163(1):27-39, 2010.

[92] George Lykotrafitis, Ares J. Rosakis, and Guruswami Ravichandran. Self-healing pulse-like shear ruptures in the laboratory. Science, 313(5794):1765-1768, 2006. 
[93] Raul Madariaga. High-frequency radiation from crack (stress drop) models of earthquake faulting. Geophysical Journal International, 51(3):625-651, 1977.

[94] Satoru Maegawa, Atsushi Suzuki, and Ken Nakano. Precursors of global slip in a longitudinal line contact under non-uniform normal loading. Tribology Letters, 38(3):313-323, Jun 2010.

[95] M. Marder. New dynamical equation for cracks. Physical Review Letters, 66:2484-2487, May 1991.

[96] C. Marone. Laboratory-derived friction laws and their application to seismic faulting. Annual Review of Earth and Planetary Sciences, 26:643-696, 1998.

[97] Gregory C. McLaskey and Brian D. Kilgore. Foreshocks during the nucleation of stick-slip instability. Journal of Geophysical Research: Solid Earth, 118(6):2982-2997, 2013.

[98] Gregory C. McLaskey, Brian D. Kilgore, and Nicholas M. Beeler. Slippulse rupture behavior on a $2 \mathrm{~m}$ granite fault. Geophysical Research Letters, 42(17):7039-7045, 2015. 2015GL065207.

[99] Michael Mello, Harsha S. Bhat, and Ares J. Rosakis. Spatiotemporal properties of sub-rayleigh and supershear rupture velocity fields: Theory and experiments. Journal of the Mechanics and Physics of Solids, 93:153 - 181, 2016.

[100] K. Nagata, M. Nakatani, and S. Yoshida. A revised rate- and statedependent friction law obtained by constraining constitutive and evolution laws separately with laboratory data. Journal of Geophysical Research: Solid Earth, 117(B2), 2012.

[101] Masao Nakatani. Conceptual and physical clarification of rate and state friction: Frictional sliding as a thermally activated rheology. Journal of Geophysical Research: Solid Earth, 106(B7):13347-13380, 2001.

[102] S. Nielsen, J. Taddeucci, and S. Vinciguerra. Experimental observation of stick-slip instability fronts. Geophysical Journal International, 180(2):697-702, 2010.

[103] Mitiyasu Ohnaka. The Physics of Rock Failure and Earthquakes. Cambridge Univ. Press, New York, 1 edition, 2013.

[104] Mitiyasu Ohnaka and Yasuto Kuwahara. Characteristic features of local breakdown near a crack-tip in the transition zone from nucleation to unstable rupture during stick-slip shear failure. Tectonophysics, 175(1):197 - 220, 1990. Earthquake Source Processes. 
[105] Mitiyasu Ohnaka and Lin-feng Shen. Scaling of the shear rupture process from nucleation to dynamic propagation: Implications of geometric irregularity of the rupturing surfaces. Journal of Geophysical Research: Solid Earth, 104(B1):817-844, 1999.

[106] Paul G. Okubo and James H. Dieterich. Fracture energy of stick-slip events in a large scale biaxial experiment. Geophysical Research Letters, 8(8):887-890, 1981.

[107] Paul G. Okubo and James H. Dieterich. Effects of physical fault properties on frictional instabilities produced on simulated faults. Journal of Geophysical Research: Solid Earth, 89(B7):5817-5827, 1984.

[108] Erik L. Olson and Richard M. Allen. The deterministic nature of earthquake rupture. Nature, 438:212 EP -, Nov 2005.

[109] Marco Paggi and Michele Ciavarella. The coefficient of proportionality between real contact area and load, with new asperity models. Wear, 268(7):1020 - 1029, 2010.

[110] A. C. Palmer and J. R. Rice. The growth of slip surfaces in the progressive failure of over-consolidated clay. Proceedings of the Royal Society of London A: Mathematical, Physical and Engineering Sciences, 332(1591):527$548,1973$.

[111] François X. Passelègue, David L. Goldsby, and Olivier Fabbri. The influence of ambient fault temperature on flash-heating phenomena. Geophysical Research Letters, 41(3):828-835, 2014.

[112] François X. Passelègue, Alexandre Schubnel, Stefan Nielsen, Harsha S. Bhat, and Raùl Madariaga. From sub-rayleigh to supershear ruptures during stick-slip experiments on crustal rocks. Science, 340(6137):12081211, 2013.

[113] Lars Pastewka and Mark O. Robbins. Contact between rough surfaces and a criterion for macroscopic adhesion. Proceedings of the National Academy of Sciences, 111(9):3298-3303, 2014.

[114] Zhigang Peng and Joan Gomberg. An integrated perspective of the continuum between earthquakes and slow-slip phenomena. Nature Geosci, 3(9):599-607, 2010.

[115] B. N. J. Persson. Theory of rubber friction and contact mechanics. The Journal of Chemical Physics, 115(8):3840-3861, 2001.

[116] Bo Persson. Sliding Friction: Physical Principles and Applications. Springer-Verlag Berlin Heidelberg, 2 edition, 2000.

[117] A. N. B. Poliakov, R. Dmowska, and J. R. Rice. Dynamic shear rupture interactions with fault bends and off-axis secondary faulting. Journal of Geophysical Research-Solid Earth, 107(B11), 2002. 
[118] Sergey G. Psakhie, Evgeny V. Shilko, Mikhail V. Popov, and Valentin L. Popov. Key role of elastic vortices in the initiation of intersonic shear cracks. Physical Review E, 91(6):063302, 2015.

[119] E Rabinowicz. The intrinsic variables affecting the stick-slip process. Proceedings of the Physical Society, 71(4):668, 1958.

[120] Ernest Rabinowicz. Friction coefficients of noble metals over a range of loads. Wear, 159(1):89 - 94, 1992.

[121] M. Radiguet, D.S. Kammer, and J.F. Molinari. The role of viscoelasticity on heterogeneous stress fields at frictional interfaces. Mechanics of Materials, 80:276 - 287, 2015. Materials and Interfaces.

[122] Mathilde Radiguet, David S. Kammer, Philippe Gillet, and Jean-François Molinari. Survival of heterogeneous stress distributions created by precursory slip at frictional interfaces. Physical Review Letters, 111:164302, Oct 2013 .

[123] Ze 'ev Reches and David A. Lockner. Fault weakening and earthquake instability by powder lubrication. Nature, 467(7314):452-455, 2010.

[124] J. R. Rice. A path independent integral and the approximate analysis of stress concentration by notches and cracks. Journal of Applied Mechanics, 35:379-386, 1968.

[125] James R. Rice. Heating and weakening of faults during earthquake slip. Journal of Geophysical Research: Solid Earth, 111, 2006. B05311.

[126] James R. Rice, Nadia Lapusta, and K. Ranjith. Rate and state dependent friction and the stability of sliding between elastically deformable solids. Journal of the Mechanics and Physics of Solids, 49(9):1865 - 1898, 2001.

[127] A. J. Rosakis, O. Samudrala, and D. Coker. Cracks faster than the shear wave speed. Science, 284(5418):1337-1340, 1999.

[128] A. M. Rubin and J.P. Ampuero. Earthquake nucleation on (aging) rate and state faults. Journal of Geophysical Research: Solid Earth, 110(B11):B11312, 2005.

[129] V. Rubino, A. J. Rosakis, and N. Lapusta. Understanding dynamic friction through spontaneously evolving laboratory earthquakes. Nature communications, 8:15991, 2017.

[130] S. M. Rubinstein, G. Cohen, and J. Fineberg. Detachment fronts and the onset of dynamic friction. Nature, 430(7003):1005-1009, 2004.

[131] S. M. Rubinstein, G. Cohen, and J. Fineberg. Dynamics of precursors to frictional sliding. Physical Review Letters, 98(22):226103, 2007. 
[132] Andy Ruina. Slip instability and state variable friction laws. Journal of Geophysical Research: Solid Earth, 88(B12):10359-10370, 1983.

[133] O. Samudrala, Y. Huang, and A. J. Rosakis. Subsonic and intersonic shear rupture of weak planes with a velocity weakening cohesive zone. Journal of Geophysical Research-Solid Earth, 107(B8), 2002.

[134] C.H Scholz. The mechanics of earthquakes and faulting. Cambridge University Press, Cambridge, 2nd edition, 2002.

[135] Hadar Shlomai and Jay Fineberg. The structure of slip-pulses and supershear ruptures driving slip in bimaterial friction. Nature Communications, 7:11787 EP -, Jun 2016. Article.

[136] Ilya Svetlizky, Elsa Bayart, Gil Cohen, and Jay Fineberg. Frictional resistance within the wake of frictional rupture fronts. Physical Review Letters, 118:234301, Jun 2017.

[137] Ilya Svetlizky and Jay Fineberg. Classical shear cracks drive the onset of dry frictional motion. Nature, 509(7499):205-208, 2014.

[138] Ilya Svetlizky, David S. Kammer, Elsa Bayart, Gil Cohen, and Jay Fineberg. Brittle fracture theory predicts the equation of motion of frictional rupture fronts. Physical Review Letters, 118:125501, Mar 2017.

[139] Ilya Svetlizky, Daniel Pino Muñoz, Mathilde Radiguet, David S. Kammer, Jean-François Molinari, and Jay Fineberg. Properties of the shear stress peak radiated ahead of rapidly accelerating rupture fronts that mediate frictional slip. Proceedings of the National Academy of Sciences, 113(3):542-547, 2016.

[140] Alessandro Taloni, Andrea Benassi, Stefan Sandfeld, and Stefano Zapperi. Scalar model for frictional precursors dynamics. Scientific Reports, 5:8086 EP -, Feb 2015. Article.

[141] Kaiwen Tian, Nitya N. Gosvami, David L. Goldsby, Yun Liu, Izabela Szlufarska, and Robert W. Carpick. Load and time dependence of interfacial chemical bond-induced friction at the nanoscale. Phys. Rev. Lett., 118:076103, Feb 2017.

[142] J. Trømborg, J. Scheibert, D. S. Amundsen, K. Thogersen, and A. MaltheSorenssen. Transition from static to kinetic friction: Insights from a $2 \mathrm{~d}$ model. Physical Review Letters, 107(7), 2011.

[143] Jørgen Kjoshagen Trømborg, Henrik Andersen Sveinsson, Julien Scheibert, Kjetil Thøgersen, David Skålid Amundsen, and Anders MaltheSørenssen. Slow slip and the transition from fast to slow fronts in the rupture of frictional interfaces. Proceedings of the National Academy of Sciences, 111(24):8764-8769, 2014. 
[144] Jørgen Kjoshagen Trømborg, Henrik Andersen Sveinsson, Kjetil Thøgersen, Julien Scheibert, and Anders Malthe-Sørenssen. Speed of fast and slow rupture fronts along frictional interfaces. Physical Review E, 92:012408, Jul 2015.

[145] Koji Uenishi and James R. Rice. Universal nucleation length for slipweakening rupture instability under nonuniform fault loading. Journal of Geophysical Research: Solid Earth, 108(B1):2042, 2003.

[146] A.I. Vakis, V.A. Yastrebov, J. Scheibert, C. Minfray, L. Nicola, D. Dini, A. Almqvist, M. Paggi, S. Lee, G. Limbert, J.F. Molinari, G. Anciaux, R. Aghababaei, S. Echeverri Restrepo, A. Papangelo, A. Cammarata, P. Nicolini, C. Putignano, G. Carbone, M. Ciavarella, S. Stupkiewicz, J. Lengiewicz, G. Costagliola, F. Bosia, R. Guarino, N.M. Pugno, and M.H. Müser. Modeling and simulation in tribology across scales: An overview. Tribology International, 2018.

[147] Robert C. Viesca. Self-similar slip instability on interfaces with rate- and state-dependent friction. Proceedings of the Royal Society of London A: Mathematical, Physical and Engineering Sciences, 472(2192), 2016.

[148] J. Weertman. Unstable slippage across a fault that separates elastic media of different elastic constants. Journal of Geophysical Research: Solid Earth, 85(B3):1455-1461, 1980.

[149] Huihui Weng and Hongfeng Yang. Seismogenic width controls aspect ratios of earthquake ruptures. Geophysical Research Letters, 44(6):2725$2732,2017$.

[150] Francis T. Wu, K. C. Thomson, and H. Kuenzler. Stick-slip propagation velocity and seismic source mechanism. Bulletin of the Seismological Society of America, 62(6):1621-1628, 1972.

[151] Kaiwen Xia, Ares J. Rosakis, and Hiroo Kanamori. Laboratory earthquakes: The sub-rayleigh-to-supershear rupture transition. Science, 303(5665):1859-1861, 2004.

[152] Kaiwen Xia, Ares J. Rosakis, Hiroo Kanamori, and James R. Rice. Laboratory earthquakes along inhomogeneous faults: Directionality and supershear. Science, 308(5722):681-684, 2005.

[153] Shiqing Xu and Yehuda Ben-Zion. Theoretical constraints on dynamic pulverization of fault zone rocks. Geophysical Journal International, 209(1):282, 2017.

[154] Shiqing Xu, Eiichi Fukuyama, Futoshi Yamashita, Kazuo Mizoguchi, Shigeru Takizawa, and Hironori Kawakata. Strain rate effect on fault slip and rupture evolution: Insight from meter-scale rock friction experiments. Tectonophysics, 2017. 
[155] G. Zheng and J. R. Rice. Conditions under which velocity-weakening friction allows a self-healing versus a cracklike mode of rupture. Bulletin of the Seismological Society of America, 88(6):1466-1483, 1998. 

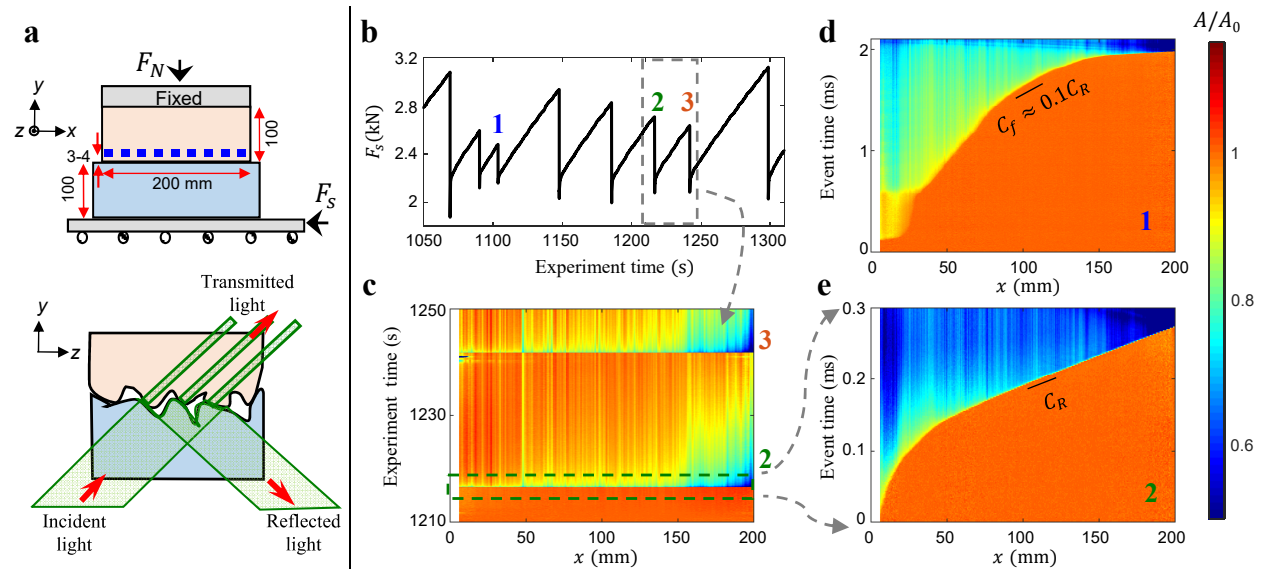

Figure 1: Stick-slip motion is mediated by propagating rupture fronts. (a) Experimental system. Two poly(methylmethacrylate) (PMMA) plates are pressed together with a normal force $F_{N}$. The real area of contact $A(x, t)$ along the $200 \mathrm{~mm}$ quasi-one-dimensional interface is measured by a method of total internal reflection (bottom) [130] at a rate of 580,000 frames per second and averaged along the $z$ direction. In parallel, the complete two-dimensional strain tensor, $\varepsilon_{i j}$, is measured along and slightly above the frictional interface at 1,000, 000 samples per second at 14-19 points along the interface (blue squares in upper figure). (b) A shear force $F_{s}$ is quasi-statically incremented during the stick phase $\left(F_{N} \approx 5500 \mathrm{~N}\right.$ and its variation is negligible). The onset of motion is characterized by rapid drops in $F_{s}$ and (c) a rapid reduction of $A(x, t)$. Note that the events 1,2 and 3 labeled in (b) correspond to those described in (c), (d) and (e). In the inter-event times $A$ re-heals due to aging $[28,100]$ and the interface regains its strength. In (c) $A(x, t)$ was normalized at the nucleation time of event 2. Color bar appears on the right. (d,e) Typical examples of the short time evolution of $A(x, t)$ (normalized by $A_{0}=A\left(x, t_{n u c}\right)$ at the time $t_{n u c}$ at which the nucleation of each event occurred) for (d) a slow and (e) a fast rupture front. Here, all ruptures nucleated at $x \approx 0$ and accelerated while propagating in the positive $x$ direction. For PMMA $C_{R} \approx 1237 \mathrm{~m} / \mathrm{s}$. 
a

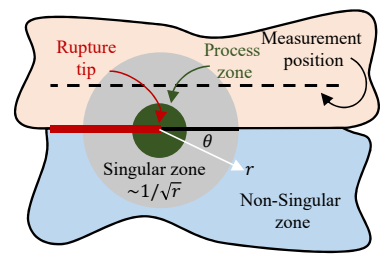

b

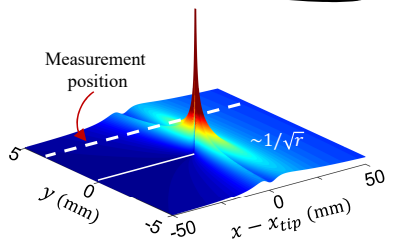

c

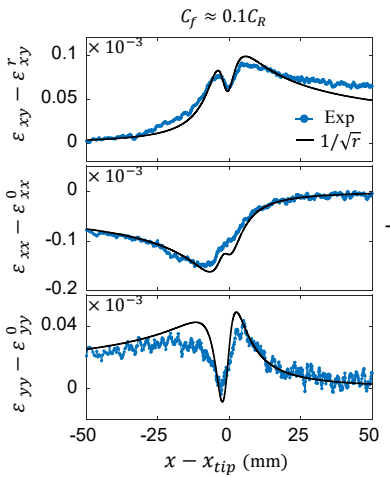

d

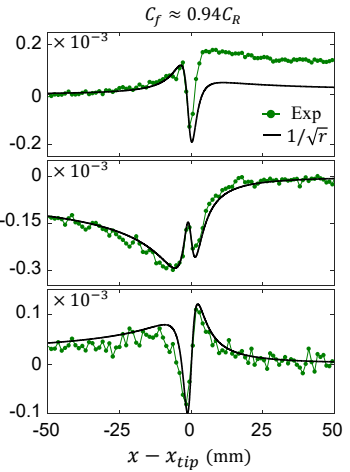

Figure 2: The $1 / \sqrt{r}$ form of the elastic strains. (a) Stresses at the vicinity of the crack tip have a universal $1 / \sqrt{r}$ singular form (gray region). Singular stresses are regularized in the process zone (green region), where dissipation takes place. Non-singular contributions should be taken into account at distances from the crack tip where they become comparable to the singular contribution. (b) The calculated shear strain $\varepsilon_{x y}$ surrounding the rupture tip predicted by equation 5 for $C_{f}=0.1 C_{R}$. The white dashed line corresponds to the strain measurement location relative to the frictional interface $y=0$. Note the angular dependence that drives the rapid oscillations evident in the measurements presented in $\mathrm{c}$ and d. Measurements of strain tensor variations, $\varepsilon_{i j}$, slightly above the frictional interface $(y=4 \mathrm{~mm})$, for (c) a slowly propagating rupture $\left(C_{f} \approx 0.1 C_{R}\right)$ and $(\mathrm{d})$ a rapid rupture $\left(C_{f} \approx 0.94 C_{R}\right)$. These measurements were acquired during the rupture events presented in Fig. 1d\&e. The corresponding LEFM predictions of Eq. 5 are plotted in black. Here both measurements were performed for the same normal load, but for different values of the imposed shear. In both, $\Gamma \approx 2.5 \mathrm{~J} / \mathrm{m}^{2}$ is the sole free parameter. $\varepsilon_{x y}^{r}, \varepsilon_{x x}^{0}$, and $\varepsilon_{y y}^{0}$ are, respectively, the residual shear strain after passage of the fronts and the initial values of the strain tensor in the propagation and normal directions. 

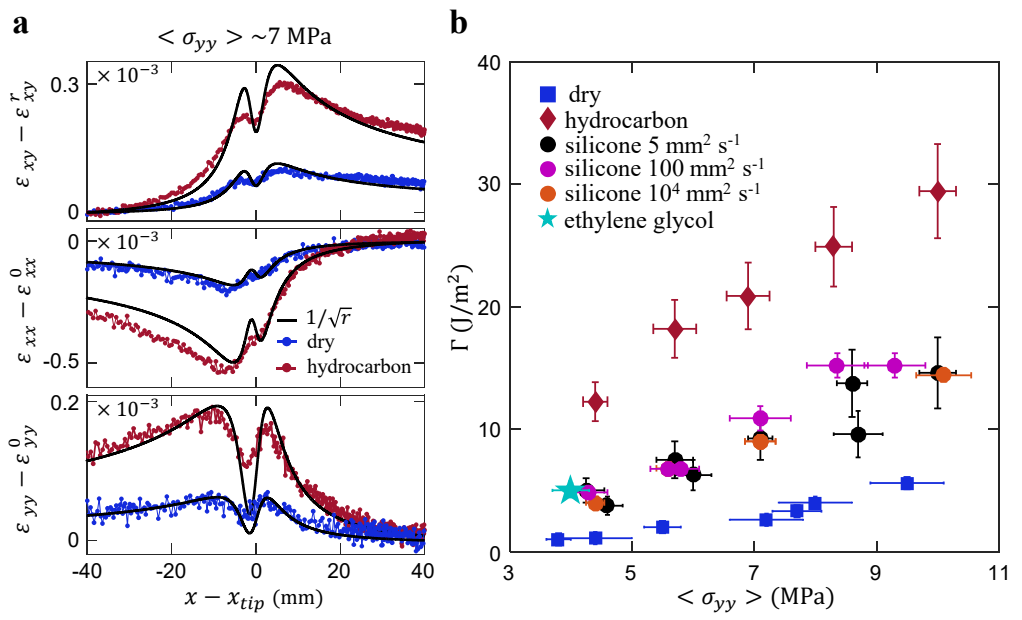

Figure 3: Dependence of the fracture energy with normal stress. (a) Comparison of $\varepsilon_{i j}$ for ruptures propagating along dry (blue line) and boundary lubricated (brown line) interfaces. In both, the applied normal stress was $<\sigma_{y y}>\sim 7 M P a$ and $C_{f} \approx 0.3 C_{R}$. Black solid lines are fits to the LEFM solution (Eq. 5). The only fitting parameter is the fracture energy; $\Gamma_{d r y}=2.6 \pm 0.3 \mathrm{~J} / \mathrm{m}^{2}$ for the dry and $\Gamma_{l u b}=23 \pm 3 \mathrm{~J} / \mathrm{m}^{2}$ for the lubricated interfaces. (b) Measured values $\Gamma$ for both the dry and lubricated interfaces versus the normal load. All $\Gamma$ vary linearly with $F_{N} ; \Gamma$ is independent of the lubricant viscosity while being highly dependent on lubricant composition. Data were taken from Ref. [23]. 

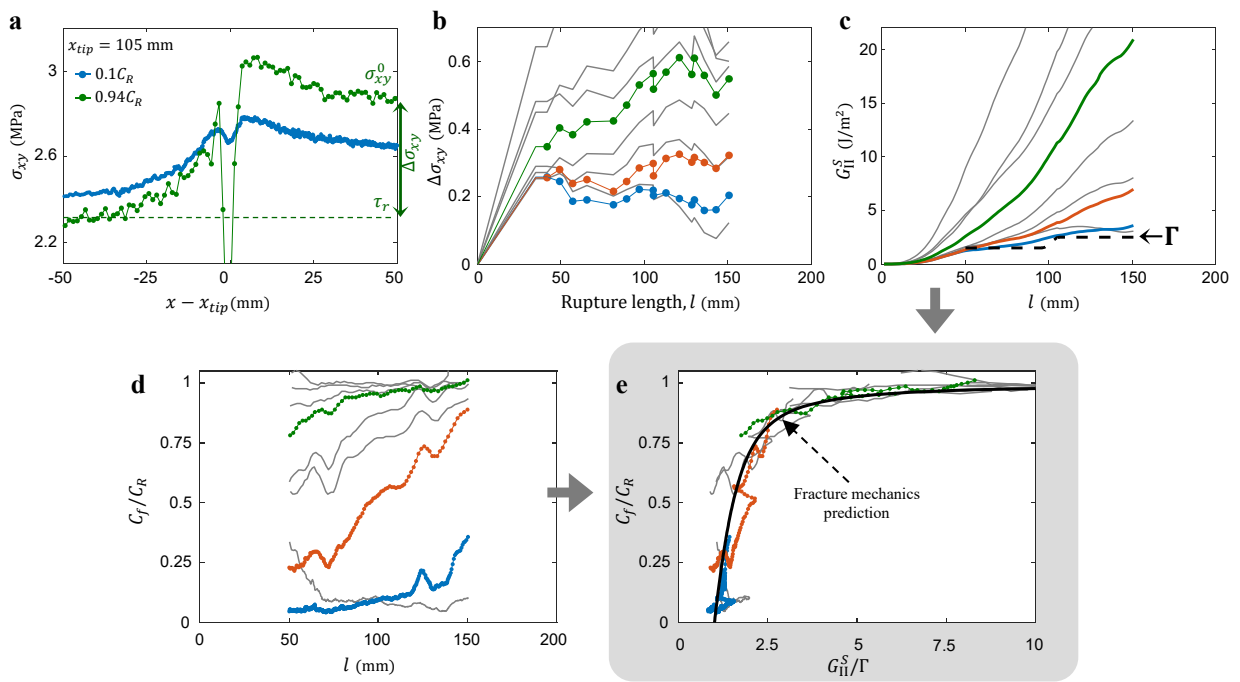

Figure 4: The equation of motion for frictional ruptures. (a) Shear stress snapshots, $\sigma_{x y}$, calculated from the measured $\varepsilon_{x y}$ presented in Fig. 2. $\sigma_{x y}^{0}$ is the initial stress level, prior to the rupture arrival, while $\tau_{r}$ denotes the residual stress measured behind the rupture tip. Their difference $\Delta \sigma_{x y}=\sigma_{x y}^{0}-\tau_{r}$ defines the dynamic stress drop. (b) Measured values of $\Delta \sigma_{x y}$ for a number of different experiments - each with the same value of applied $F_{N} . \Delta \sigma_{x y}$ near $x=0$ are extrapolated to $\Delta \sigma_{x y}=0$ at $x=0$ with (d) their corresponding rupture velocity profiles $C_{f}(l)$. (c) The $\Delta \sigma_{x y}$ profiles in (b) yield static energy release rates, $G_{\mathrm{II}}^{S}\left(l, \Delta \sigma_{x y}\right)$. Dashed line - the measured fracture energy profile, $\Gamma(l)$. Slow rupture fronts (blue) are associated with $G_{\mathrm{II}}^{S} \approx \Gamma$ while ruptures rapidly accelerate towards $C_{R}$ (green) once $G_{\mathrm{II}}^{S} \gg \Gamma$. (e) All of the $C_{f}(l)$ profiles collapse to a single functional form $C_{f}\left(G_{\mathrm{II}}^{S} / \Gamma\right)$, given by the classical equation of motion for shear cracks, Eq. 6 (black line). The blue and green examples correspond to the slow and fast events in Fig. 1d\&e and Fig. 2c\&d. $l$ - rupture length. Data were taken from Ref. [138]. 


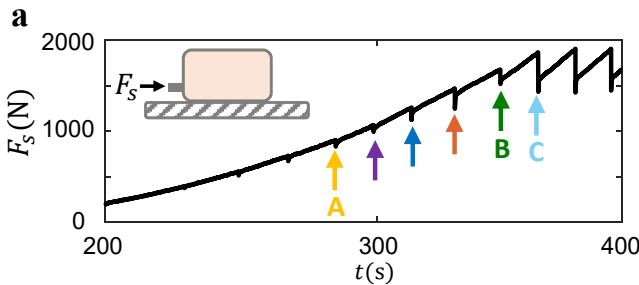

c

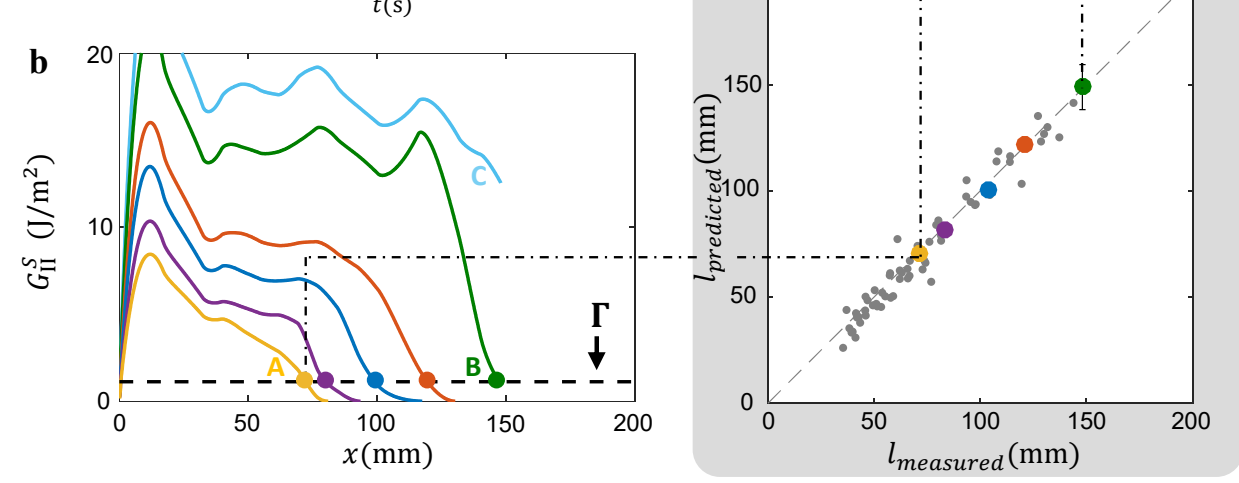

Figure 5: Prediction of rupture arrest locations. (a) Loading conditions characterized by $F_{S}$ applied locally (inset) are considered. Well before global sliding (event $\mathrm{C}$ ) a sequence of small force drops are observed. (b) The computed static energy release rate $G_{\mathrm{II}}^{S}$ along $x$ for the slip events in (a). Circles denote the predicted location of the arrest, $x=l_{\text {predicted }}$, as determined by $G_{\mathrm{II}}^{S}=\Gamma$ ( $\Gamma$ is denoted by the dashed line). Note that for event $\mathrm{C}$, the first system-wide sliding event, $G_{\mathrm{II}}^{S}>\Gamma$. (c) Comparison of the measured arrested rupture lengths, $l_{\text {measured }}$, as determined from the contact area measurements to the predicted lengths, $l_{\text {predicted }}$ computed in b. 12 additional experiments, each with different normal loads, fracture energies and stress distributions are given by the gray dots. The dashed line has a slope of 1. Data were taken from Ref. [25]. 

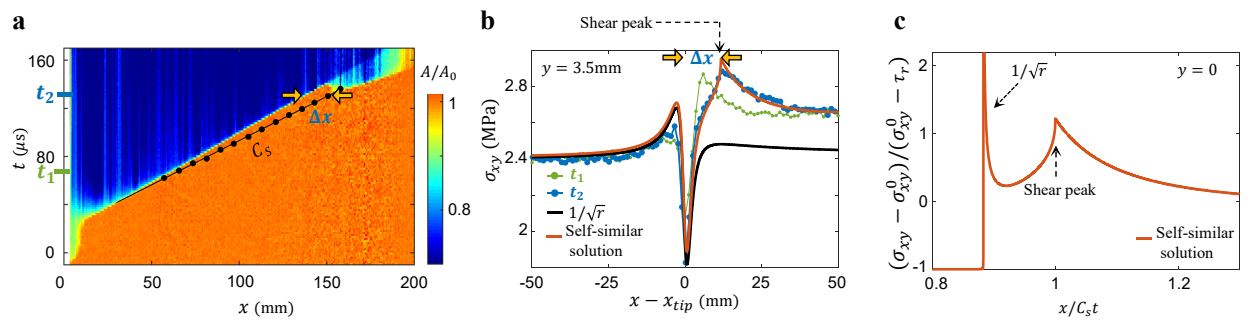

Figure 6: Stress wave radiation of accelerating ruptures. (a) $A(x, t)$ evolution (normalized at nucleation time, $t=0$ ), along the quasi-1D interface due to a rupture front that nucleated at $x \approx 0$, rapidly accelerated to $C_{R}$, and transitioned to supershear at $x \approx 155 \mathrm{~mm}$. (b) $\sigma_{x y}$ relative to the rupture tip position $x_{t i p}$ at the two instances, $t_{1}$ (green) and $t_{2}$ (blue) denoted in (a), show prominent amplitude shear strain peaks preceding the rupture tip arrival. Successive measurements (black points in (a)) reveal that these peaks propagate at $C_{S}$, and trigger supershear rupture. Measurements at $t_{2}$ are compared with both the singular LEFM predictions and the self-similar solution. While the singular prediction fails at $x-x_{t i p}>0$ the self-similar solution entirely captures the measurements including the initial shear strain $\sigma_{x y}^{0}$ and the shear stress peak far before the rupture tip arrival. (c) Close-up of the self-similar solution (see text), at $y=0$ shows a pronounced shear stress peak propagating ahead of the singular rupture tip at $C_{S}$. Data were taken from Ref. [139]. 

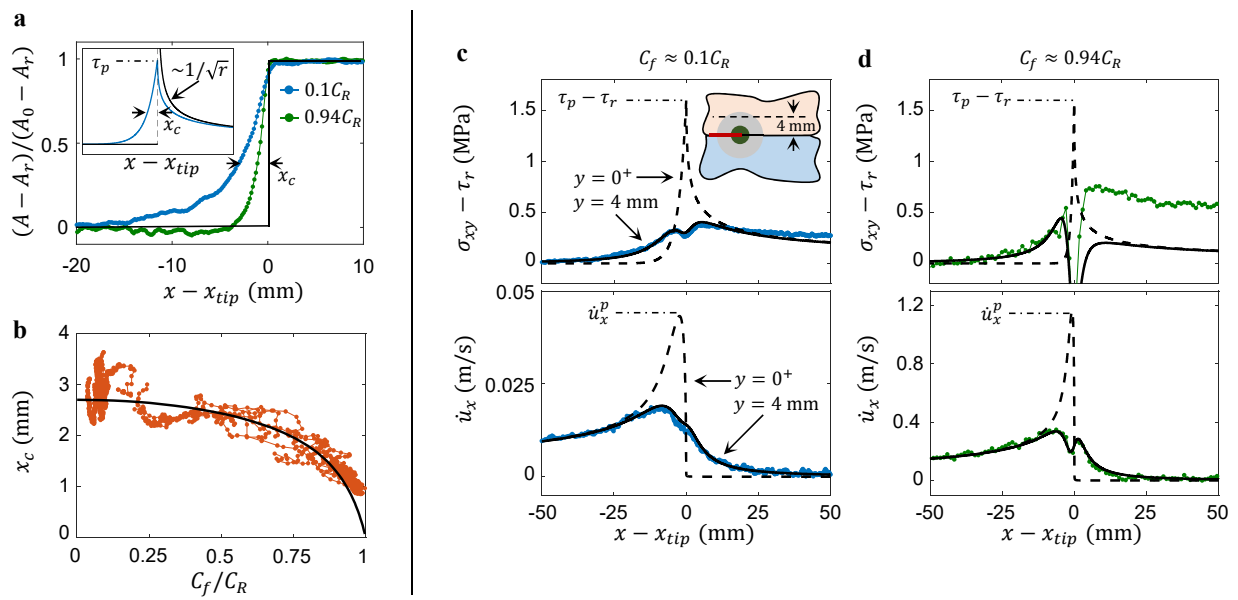

Figure 7: Regularization of the elastic singular fields at the rupture tip. (a) The normalized real area of contact, $A$, is plotted relative to the rupture tip position for the two rupture events described in Fig. 1d\&e and Fig. 2. $A_{0}$ and $A_{r}$ are the initial (prior to rupture arrival) and residual (after rupture passage) values of $A$, respectively. $x_{c}$ is the length scale corresponding to a $60 \%$ reduction of $A$ and represents the scale at which the singular fields are regularized. Note the contraction of $x_{c}$ with increasing $C_{f}$. (inset) Schematic drawing of a nonsingular cohesive zone model in which the shear stress is reduced exponentially behind the crack tip once the peak strength, $\tau_{p}$, is reached (blue line). Far ahead of the crack tip the solution matches the square-root singular form (black line). (b) $x_{c}$ contracts as $C_{f} \rightarrow C_{R}$. Black line - LEFM prediction. (c,d) Measurements of the shear stress variation (top) and particle velocity $\dot{u}_{x}=-\varepsilon_{x x} C_{f}$ [137] at the measurement plane located $4 \mathrm{~mm}$ above the interface for (c) slow and (d) rapid ruptures. The exponential cohesive zone model at the interface is defined by two parameters, $\Gamma$ and $x_{c} . x_{c}$ is estimated from measurements of $A$ in (a). $\Gamma$ was measured in Fig. 2. Interface (dashed lines) and off-interface (solid lines) predictions of the cohesive zone model. 